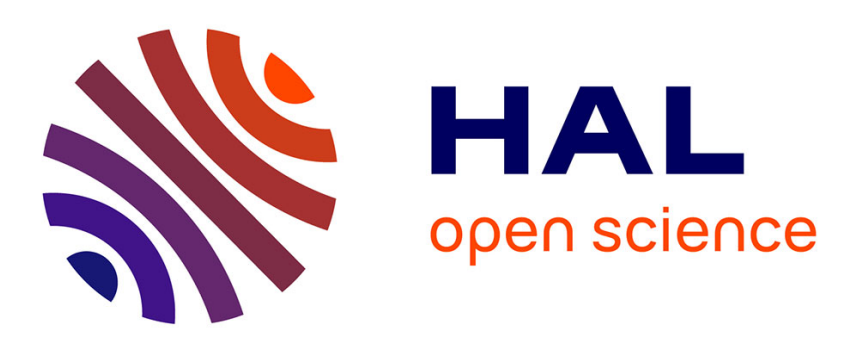

\title{
Ressources florales exploitées par quelques Apoïdes des zones cultivées en savane arborée sénégalaise durant la saison des pluies
}

Danielle Lobreau-Callen, Remi Coutin

\section{- To cite this version:}

Danielle Lobreau-Callen, Remi Coutin. Ressources florales exploitées par quelques Apoïdes des zones cultivées en savane arborée sénégalaise durant la saison des pluies. Agronomie, 1987, 7 (4), pp.231-246. hal-00884988

\section{HAL Id: hal-00884988 \\ https://hal.science/hal-00884988}

Submitted on 1 Jan 1987

HAL is a multi-disciplinary open access archive for the deposit and dissemination of scientific research documents, whether they are published or not. The documents may come from teaching and research institutions in France or abroad, or from public or private research centers.
L'archive ouverte pluridisciplinaire HAL, est destinée au dépôt et à la diffusion de documents scientifiques de niveau recherche, publiés ou non, émanant des établissements d'enseignement et de recherche français ou étrangers, des laboratoires publics ou privés. 


\title{
Ressources florales exploitées par quelques Apoïdes des zones cultivées en savane arborée sénégalaise durant la saison des pluies
}

\author{
Danielle LOBREAU-CALLEN \& Remi COUTIN (*) \\ C.N.R.S., Laboratoire de Phanérogamie du Muséum et Laboratoire de Phytomorphologie de l'E.P.H.E., \\ Muséum national d'Histoire naturelle, 16, rue Buffon, F 75005 Paris. \\ (*) I.N.R.A., Station de Zoologie, Route de Saint-Cyr, F 78000 Versailles.
}

RÉSUMÉ

Au Sénégal, en zone soudanienne, la saison des pluies s'étend sur un peu plus de 3 mois, de telle sorte que de nombreux végétaux spontanés et cultivés présentent une floraison groupée sur un court laps de temps ; ceux étudiés correspondent à 5 types de groupements bien définis.

Quinze espèces d'Apoïdes appartenant aux genres Xylocopa spp., Anthophora s. 1., Megachile s. 1. et Tetralonia sp. capturées en cours de butinage sur un petit nombre de plantes, ont fait l'objet d'une étude comparative afin de déterminer si elles effectuent un choix qui tient compte : du phénotype de la plante, de la morphologie florale ou pollinique ou des deux ensemble. Les groupements végétaux dans lesquels ces insectes ont butiné sont également pris en considération.

Le comportement de collecte est fort différent selon que l'individu visite une fleur pour se nourrir en ingérant du pollen, ou pour ramasser ce dernier sur ses pattes postérieures ou sa brosse ventrale, et constituer peu à peu dans chaque cellule la réserve alin. sntaire ou «pain d'abeille » de la larve.

L'usure des poils du revêtement dors ' 1 du mésothorax, en accroissant très fortement la rétention des grains de pollen est cependant un facteur défavorable à la pollinisation croisée des fleurs visitées.

Mots clés additionnels : Interrelations Plantes/Apoïdes solitaires, pollens collectés, Anthophora s. l., Megachile $s . l$., Xylocopa $s . l$. during the rainy season.

In the sudanian zone of Senegal the rainy season lasts a little more than 3 months, so that plenty of wild and cultivated plants flower together over a short space of time; we studied species belonging to 5 well defined groups. About fifteen species of Apoidea from the 4 genera Xylocopa spp., Anthophora s. 1., Megachile s. 1. and Tetralonia sp., captured during foraging activity, were studied to determine if they selected flowers according to floral or pollen morphology, or both. The vegetation types in which these insects foraged were also taken into consideration. Foraging behaviour was very different according to whether the insect visited a flower for feeding purposes, when the pollen is swallowed, or for gathering larval food-pollen in the corbicula on their hind-legs or on the ventral brush, to be deposited in each nest cell. The wear of the dorsal hairs of the mesothorax, while strongly favouring the retention of pollen grains, was nevertheless an unfavourable factor as regards the cross-pollination of the flowers visited.

Additional key words : Host-plant/solitary bee interrelations, collected pollen, Anthophora s. l. (diggerbees), Megachile $s$. l. (leafcutting bees), Xylocopa s. l. (carpenter bees).

\section{INTRODUCTION}

Les rapports mutualistes existant entre les plantes et les insectes, où chacun des partenaires tire réciproquement des bénéfices, commencent à être bien connus, du moins dans les régions tempérées. C'est le cas des "mécanismes de la pollinisation entomogame chez la plante et de récolte de pollen chez l'insecte, en particu- lier chez les Hyménoptères Apoïdes » (GILLON, 1986), les femelles de ces derniers étant souvent inféodées à certaines plantes pour l'alimentation de leurs larves.

Des exemples de " coévolution cumulative " (GILLON, 1986) plantes/hyménoptères dans lesquels deux cladogrammes parallèles ont pu être mis en évidence ont été décrits ; mais ils sont très peu nombreux pour les régions tropicales. Il s'agit par exemple des interre- 
lations Ficus (Moraceae)/Agaonidae (Hyménoptères) (LACHAISE, 1982, BAIJNATH \& RAMCHARUM, 1983, JERMY, 1984), Krameriaceae, Malpighiaceae/Anthophoridae (LOBREAU-CALLEN, 1983a, b).

Les phénomènes de relations mutualistes plantes/abeilles, se traduisant par une simple coadaptation, sont les plus fréquents et parfaitement bien cernés pour les régions tempérées (MCGREGOR, 1976, TASEI, 1977), plus rares pour les pays tropicaux (BUCHMAN, 1983, HURD, 1978, Vogel, 1974). Pour l'Afrique, elles sont pratiquement inexistantes. Ces rapports sont parfois saisonniers comme c'est le cas des Malpighiaceae des régions semi-arides dont les floraisons chasmogames allogames ne se produisent que durant la saison d'envol et de grande activité des abeilles pollinisatrices spécialisées. Pendant toute la durée des stades larvaires et de la nymphose, les fleurs sont toutes cleistogames.

Des analyses polliniques ont été entreprises dans le but de mettre en évidence les interrelations coadaptatives, voire même coévolutives susceptibles d'exister entre quelques abeilles solitaires du Sénégal à la saison où elles sont les plus actives, et les plantes qu'elles butinent.

En outre, l'évaluation du niveau évolutif des taxons choisis pour le butinage dans l'ensemble de la classification phylétique des Angiospermes est essentielle, du fait notamment de la correspondance existant entre la morphologie florale et la composition chimique des nectars (BAKER \& BAKER, 1983, SOUTHWICK et al., 1981).

Une telle étude contribue également à mettre en évidence l'action pollinisatrice ou phytophage, selon les cas, des abeilles sur la dynamique de la végétation. Il est d'ailleurs déjà bien connu que de nombreuses abeilles solitaires butinent en priorité les floraisons de la canopée ou celle des arbres les plus hauts, en forêt sèche ou en savane arborée au Costa Rica (FRANKIE, 1975 ; FRANKIE et al., 1983 ; FRANKIE \& HABER, 1983).

\section{MÉTHODES ET TECHNIQUES}

\section{A. Matériel et méthodes}

Les Apoïdes qui font l'objet de cette étude appartiennent à 3 espèces du genre $X y$ locopa : $X$. olivacea F., $X$. pubescens Spin. et $X$. inconstans Smith. ; à 9 espèces du genre Megachile (sens large) : Amegachile geoffreyi Ckll., Eurymella eurymera Sm., E. tetracantha Cock., Eutricharaea azarica Ckll., E. concinna Sm., Paracella semivenusta Ckll., Neglectella stefenelii Friese, Platysta platystoma Post., Pl. sanguinea ; à 3 espèces d'Anthophora (sens large) : Anthophora nubisa Lep., Amegelia torrida Sm., Amegelia sp. et à un Tetralonia sp.

Ces insectes ont tous été capturés en cours de butinage durant les mois de septembre à novembre, pendant 4 années, de 1966 à 1970, afin de pallier aux décalages saisonniers exceptionnels. Le choix de cette saison qui correspond à la seconde moitié et à la fin de la saison des pluies, se justifie par la grande activité de ces insectes approvisionnant les cellules larvaires. Les heures où ces abeilles sont les plus affairées étant les plus chaudes de la journée, c'est donc entre 14 et $18 \mathrm{~h}$ qu'elles ont été capturées, asphyxiées à l'acétate d'éthyle et mises en collection.

Les plantes sur lesquelles les Apoïdes butinaient ont été photographiées ou récoltées pour identification et miseś en herbier. Leur identification a été assurée par H. MERLIER (CIRAD) et par J. P. LEBRUN (IMVT, Maisons-Alfort).

Parmi les 800 Apoïdes femelles capturés, seulement une centaine d'entre eux portent des pelotes permettant d'en analyser les pollens. L'exine du pollen étant parfaitement bien conservée du fait de sa structure, l'identification de ce dernier est toujours possible, même si plusieurs années séparent capture et identification.

Le pollen amassé par chaque insecte soit sur les brosses collectrices du métatarse des pattes postérieurs (Xylocopa, Anthophora), soit sur la brosse abdominale (Megachile), a été prélevé directement sur ces insectes.

Pour une meilleure étude du comportement des insectes vis-à-vis de la végétation, il est nécessaire d'établir la distinction entre les plantes butinées pour la confection des rations alimentaires du couvain et celles visitées pour l'alimentation des adultes. Pour cela, certains insectes, 1 ou 2 par espèce, ont été disséqués après ramollissement dans l'eau. Le tractus digestif, thoracique d'une part et abdominal d'autre part ont été traités séparément pour isoler le pollen et l'analyser.

\section{B. Techniques}

Après avoir été préalablement hydratés avec une goutte d'eau, directement sur la brosse collectrice de la patte gauche, parfois sur les 2 pattes pour comparaison, les pollens ont été prélevés à l'aide d'un tube capillaire, puis rincés à l'acide acétique, enfin acétolysés et rincés à l'eau soit sur lame, soit dans un tube à centrifuger, selon les techniques modifiées d'ERDTMAN, 1952, HOU, 1969, DYER \& SKINN, 1978 et Lobreau-CALlen \& CALlen, 1982.

Pour établir les proportions des divers taxons botaniques, les comptages ont porté sur 250 à 600 grains de telle sorte que tous les types polliniques aient pu être rencontrés.

Les pollens ingérés ont également été traités selon la méthode d'Erdtman, puis, si nécessaire, par une solution de potasse à 5 ou 10 p. 100, ou encore de papaïne, solvant des protéines.

\section{ANALYSE DES RÉSULTATS ET DISCUSSION}

\section{A. Résultats}

Les résultats de la centaine d'analyses polliniques sont résumés dans plusieurs tableaux complémentaires. Le tableau 1 donne le pourcentage de pollens rencontrés pour chaque espèce. Lorsque ce dernier est inférieur à $0,3 \%$, il est indiqué par $\varepsilon$. Seules les récoltes polliniques de Tetralonia ne figurent pas dans les tableaux. 
TABLEAU $1 \mathrm{~A}$

Résultats synthétiques des analyses polliniques des pelotes portées par la patte arrière gauche des Anthophora s. $l$.

Synthetic results of the pollen analysis of the corbicula on the hind-legs of Anthophora s. $l$.

\begin{tabular}{|c|c|c|c|c|}
\hline & 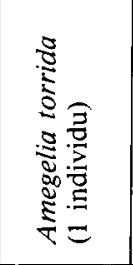 & 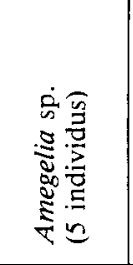 & 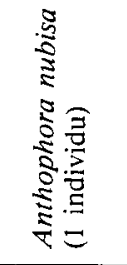 & 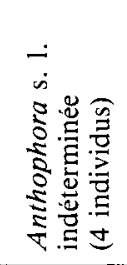 \\
\hline & $a \quad b \quad c c c$ & $\begin{array}{llll}a & b & c & d\end{array}$ & $a b c d$ & $a \quad b \quad c \quad d$ \\
\hline $\begin{array}{l}\text { Acanthaceae } \\
\text { Hygrophila senegalensis } \\
\text { Apocynaceae } \\
\text { Thevetia neriffolia } \\
\text { Asteraceae } \\
\text { Centaurea senegalensis } \\
\text { Helianthus annuus } \\
\text { Caesalpiniaceae } \\
\text { Cassia spp. } \\
\text { Convolvulaceae } \\
\text { Ipomoea dont } \text { I. batatas } \\
\text { Cucurbitaceae } \\
\text { Cucumis sp. } \\
\text { Malvaceae } \\
\text { Pavonia sp. } \\
\text { Papilionaceae } \\
\text { Tephrosia purpurea } \\
\text { Pedaliaceae } \\
\text { Sesamum alatum } \\
\text { Portulacaceae } \\
\text { Portulaca sp. } \\
\text { Rubiaceae } \\
\text { Mitracarpus villosus } \\
\text { Kauhotia grandiflora } \\
\text { Verbenaceae } \\
\text { Lantana camara }\end{array}$ & 1 & $\begin{array}{l}1 \\
1 \\
1 \\
1\end{array}$ & 1 & 1 \\
\hline
\end{tabular}

Nombre d'échantillons portant : $\varepsilon \leqslant \mathrm{a}<25 \%, 25 \leqslant \mathrm{~b}<50 \%, 50 \leqslant \mathrm{c}<75 \%$, $75 \leqslant \mathrm{~d} \leqslant 100 \%$ de pollen de chaque espèce déterminée.

Number of samples with : $\varepsilon \leqslant \mathrm{a}<25 \%, \quad 25 \leqslant \mathrm{~b}<50 \%, \quad 50 \leqslant \mathrm{c}<75 \%$, $75 \leqslant \mathrm{~d} \leqslant 100 \%$ of pollen grains of each determined species.

$\varepsilon$ indique que le pourcentage en pollen est inférieur à $0,3 \%$.

$\varepsilon \%$ pollen less than $0.3 \%$.

Dans les tableaux suivants, les caractères morphologiques et floraux des espèces butinées ainsi que les groupements végétaux auxquels ils appartiennent sont résumés. Figurent également les probabilités de pollinisation des abeilles pour ces fleurs après analyse comparative de la morphologie florale et de celle des insectes.

Les caractères polliniques sont illustrés par les figures 1 à 29. La morphologie des divers types de poils des insectes sur lesquels le pollen est déposé lors du butinage est représenté dans les figures 1 à 6 .

\section{B. Discussion}

\section{Comportement des Apoïdes}

Lors de leur activité de récolte, les Apoïdes brossent ou secouent violemment les anthères de telle sorte que plusieurs grains s'attachent à l'abdomen et parfois sur le dos de l'insecte (FAEGRI \& VAN DeR PIJL, 1979).

La collecte du pollen et sa rétention sur les corbicules des pattes ou des brosses ventrales sont assurées par des poils allongés et chevronnés; certains élargis et spatulés à leur extrémité formant arrêtoir (fig. 1, 2, 5, 6). Ces derniers retiennent fortement le pollen qui y est projeté activement par l'insecte. L'adhérence du pollen est renforcée par la présence de " pollen-coat », substance glyco-lipo-protéinique située à la surface ou dans les espaces libres de l'exine.

Au cours de la visite des fleurs, le dépôt du pollen sur le dos est passif, plus ou moins important selon l'âge de l'insecte. Lorsque les poils d'aspect plumeux qui recouvrent la partie dorsale de la tête et les sclérites thoraciques sont entiers, en particulier chez l'insecte jeune, le pollen qui s'y dépose n'est pas retenu prisonnier (fig. 3). La présence de quelques grains provient simplement du frôlement des étamines par le dos de l'insecte ou d'un phénomène électrostatique, la plante étant chargée négativement et l'abeille positivement lorsqu'elle est en mouvement (ERICKSON \& BUCHMAN, 1983). Lorsque ces poils sont usés et cassés chez les insectes âgés, le pollen est fortement fixé et comme collé en une seule couche directement sur les sclérites (fig. 4). 
TABLEAU 1B

Résultats synthétiques des analyses polliniques des pelotes de la brosse abdominale de Megachile $s . l$. Synthetic results of the pollen analysis of the corbicula on the ventral brush of Megachile $s . l$.

\begin{tabular}{|c|c|c|c|c|c|c|c|c|c|}
\hline & 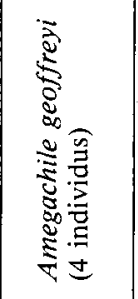 & 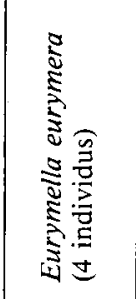 & 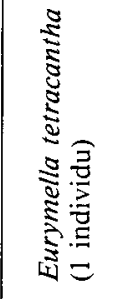 & 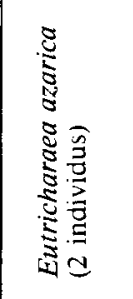 & 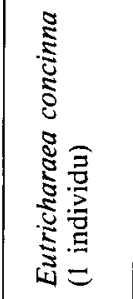 & 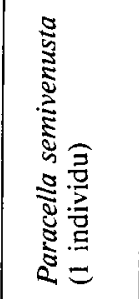 & 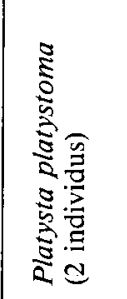 & 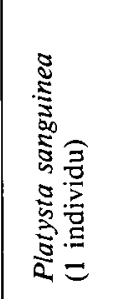 & 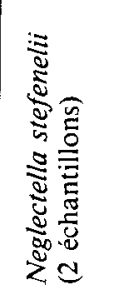 \\
\hline & $\begin{array}{llll}a & b & c & d\end{array}$ & $\begin{array}{llll}a & b & c & d\end{array}$ & $\begin{array}{llll}a & b & c & d\end{array}$ & $\begin{array}{llll}a & b & c & d\end{array}$ & $\begin{array}{llll}a & b & c & d\end{array}$ & $a \quad b \quad c c c$ & $\begin{array}{llll}a & b & c & d\end{array}$ & $\begin{array}{llll}a & b & c & d\end{array}$ & $\begin{array}{llll}a & b & c & d\end{array}$ \\
\hline $\begin{array}{l}\text { Acanthaceae } \\
\text { Justicia striata } \text { s. I. } \\
\text { Convolvulaceae } \\
\text { Ipomoea (dont } \text { I. batatas) } \\
\text { Lamiaceae } \\
\text { Ocimum cf. basilicum } \\
\text { Linaceae } \\
\text { Hugonia planchonii } \\
\text { Papilionaceae } \\
\text { Sesbania pachycarpa } \\
\text { Tephrosia spp. } \\
\text { Indigofera sp. } \\
\text { Crotalaria sp. } \\
\text { Alysicarpus sp. } \\
\text { cf. Ormocarpum } \\
\text { Tiliaceae } \\
\text { Corchorus olitorius } \\
\text { Zygophyllaceae } \\
\text { Fagonia cretica }\end{array}$ & $\begin{array}{ll}1 & 3 \\
1 & \end{array}$ & 1 & $\begin{array}{l}1 \\
1\end{array}$ & 1 & 1 & $\begin{array}{llll}1 & & & \\
& & & 1 \\
& 1 & & \\
& 1 & & \end{array}$ & $\begin{array}{l}1 \\
1 \\
1\end{array}$ & 1 & $\begin{array}{ll}1 & 1 \\
& 1\end{array}$ \\
\hline
\end{tabular}

Nombre d'échantillons portant : $\varepsilon \leqslant \mathrm{a}<25 \%, 25 \leqslant \mathrm{~b}<50 \%, 50 \leqslant \mathrm{c}<75 \%, 75 \leqslant \mathrm{~d} \leqslant 100 \%$ de pollen de chaque espèce déterminée. Number of samples with : $\varepsilon \leqslant \mathrm{a}<25 \%, 25 \leqslant \mathrm{~b}<50 \%, 50 \leqslant \mathrm{c}<75 \%, 75 \leqslant \mathrm{~d} \leqslant 100 \%$ of pollen grains of each determined species. $\varepsilon$ indique que le pourcentage en pollen est inférieur à $0,3 \%$.

$\varepsilon \%$ pollen less than $0.3 \%$.

Ainsi, l'intense activité déployée par une abeille " jeune " pour l'approvisionnement de ses cellules aurait pour conséquence un transport plus actif du pollen de plante à plante. C'est par exemple le cas de l'Ocimum basilicum $\mathrm{L}$. visité par la mégachile $P$. platystoma ; c'est aussi celui de Sesamum sp. visité principalement par Anthophora sp.

Il est évident que dans le cas des individus les plus jeunes, lors du contact du dos avec les stigmates, les grains de pollen posés à la surface des poils ramifiés et non adhérents sont plus facilement recueillis par le stigmate. En revanche, dans le cas des insectes âgés, le pollen fortement fixé aux sclérites est difficilement repris par les stigmates et l'abeille serait alors une pollinisatrice moins efficace.

Lorsque les styles sont courts, sessiles ou mêlés aux étamines, la pollinisation se fait par frottement des stigmates sur la face ventrale de l'abeille, ce qui correspond toujours à un comportement de collecte intense de pollen pour l'approvisionnement de la cellule (ex. Megachile). Là encore, les insectes « jeunes » seraient les pollinisateurs les plus efficaces en raison de leur intense activité de récolte.

\section{Végétaux visités sur les Apoides d'après la composi- tion pollinique des pelotes}

Parmi les Apoïdes étudiés, certains marquent une préférence pour un taxon botanique défini. C'est ainsi que le genre Tetralonia est monolectique. Il est le principal pollinisateur des espèces d'Ipomoea (Convolvulaceae). L'individu étudié n'avait récolté du pollen que sur Ipomoea batatas (L.) Lam., cultivé dans la région.

Les 3 autres genres : Xylocopa, Anthophora, Megachile s. 1., sont polylectiques (tabl. 1) quelles que soient les dimensions des insectes. Au cours d'un seul voyage, ils amassent habituellement le pollen d'une à 4 espèces botaniques différentes, parfois davantage, jusqu'à 11 .

Chez les espèces de grande taille comme Xylocopa inconstans, il n'existe pas de relation entre la taille de l'insecte et celle du pollen. C'est ainsi que Paracella semivenusta qui mesure $35 \mathrm{~mm}$ de long a récolté du pollen d'Alysicarpus sp. (50 $\mu \mathrm{m}$ environ) (fig. 16) en mélange avec des pollens d'Ormocarpum sp. $(15 \mu \mathrm{m})$ (fig. 18), de Crotalaria sp. $(19 \mu \mathrm{m})$ (fig. 7) et de Justicia sp. $(32 \mu \mathrm{m})($ fig. 8,9$)$.

Les pollens des fleurs sur lesquelles les Anthophora et les Megachile ont été capturées, sont toujours retrouvés, au cours de l'analyse, fixés aux brosses tarsales ou ventrales de ces Apoïdes : par exemple, les pollens d'Hygrophila senegalensis (Nees) Anders (fig. 26), de Lantana camara L., de Mitracarpus villosus (Sw.) DC (fig. 29), de Sesbania sp. (fig. 17), de Tephrosia sp. et de Thevetia neriifolia Juss. pour les anthophores, ou de Sesbania pachycarpa (fig. 5) et de Crotalaria sp. pour les mégachiles. En revanche, les pollens des fleurs sur lesquelles les xylocopes ont été capturés ne sont pas nécessairement retrouvés sur les 
TABLEAU IC

Résultats synthétiques des analyses polliniques des pelotes portées par la patte postérieure gauche des Xylocopa.

Synthetic results of the pollen analysis of the corbicula on the left hind-legs of Xylocopa.

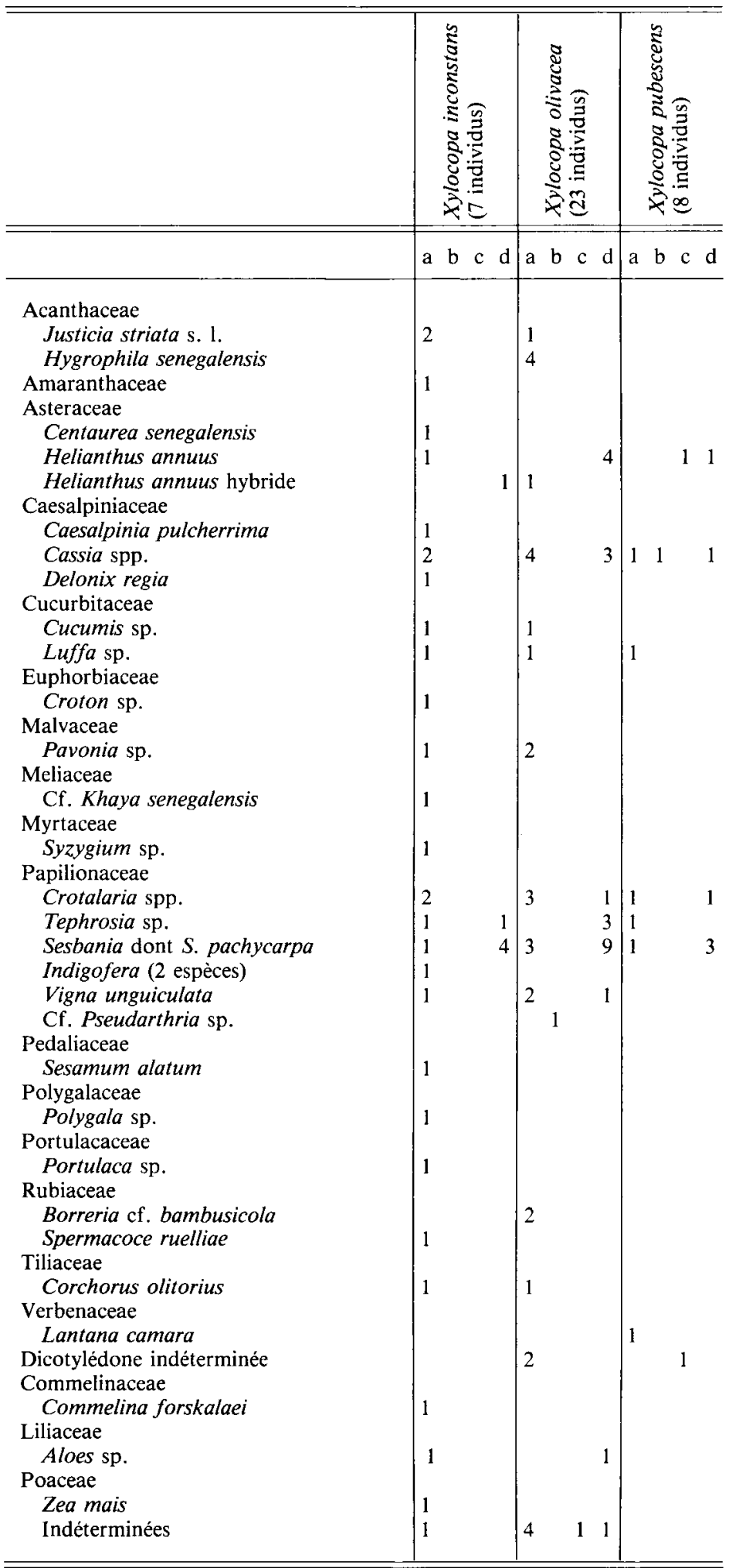

Nombre d'échantillons portant: $\varepsilon \leqslant \mathrm{a}<25 \%, 25 \leqslant \mathrm{~b}<50 \%$, $50 \leqslant \mathrm{c}<75 \%, 75 \leqslant \mathrm{~d} \leqslant 100 \%$ de pollen de chaque espèce déterminée. Number of samples with : $\varepsilon \leqslant \mathrm{a}<25 \%, 25 \leqslant \mathrm{~b}<50 \%, 50 \leqslant \mathrm{c}<.75 \%$, $75 \leqslant \mathrm{~d} \leqslant 100 \%$ of pollen grains of each determined species.

$\varepsilon$ indique que le pourcentage en pollen de l'espèce considérée est inférieur à $0,3 \%$.

$\varepsilon \%$ pollen grains of the considered species less than $0.3 \%$. 

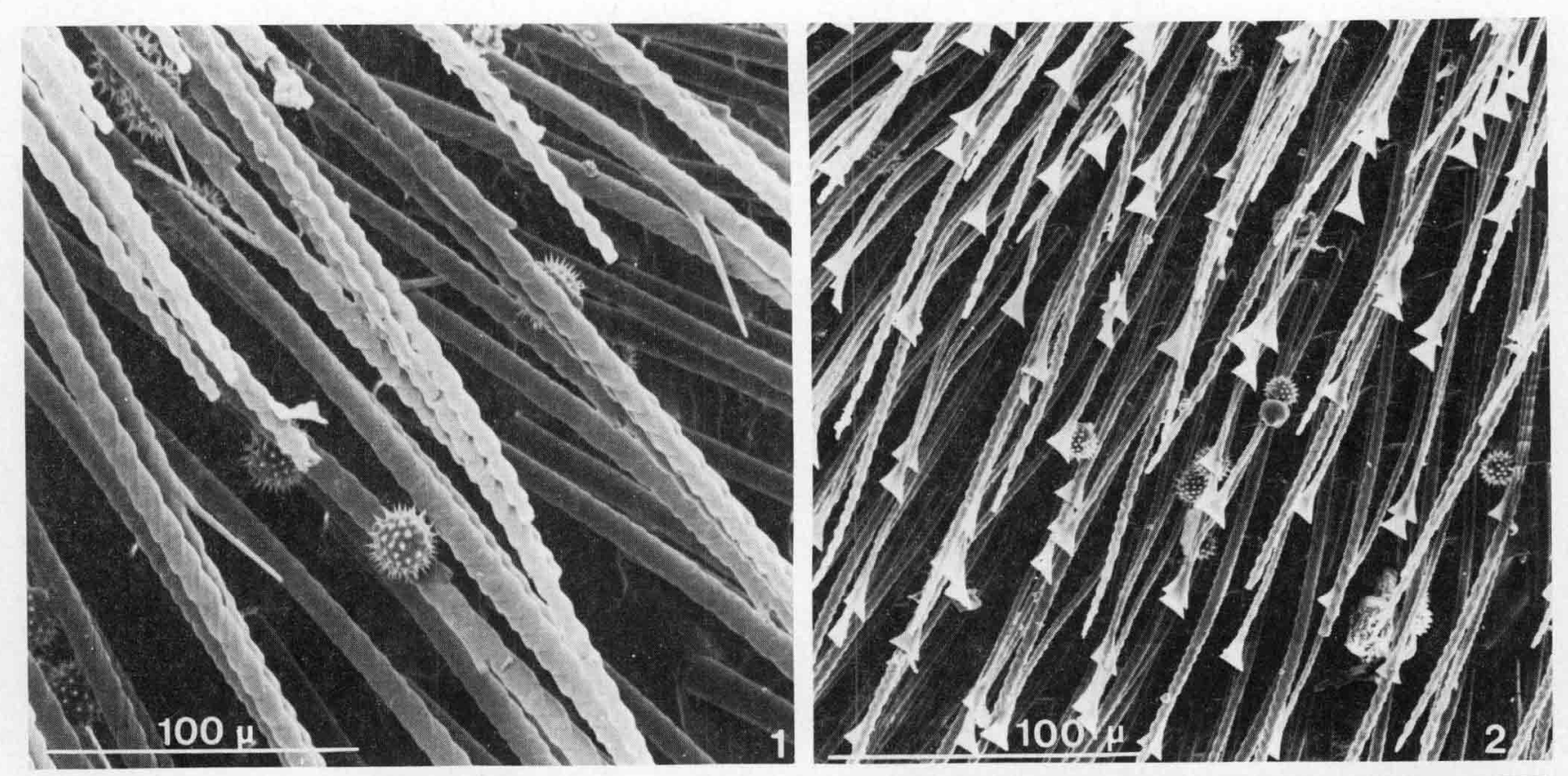

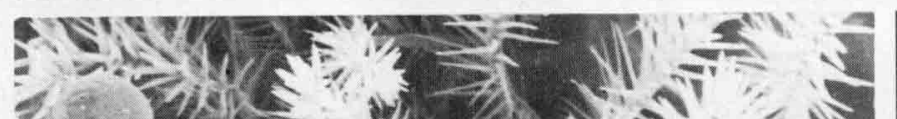
ma $=y, y$

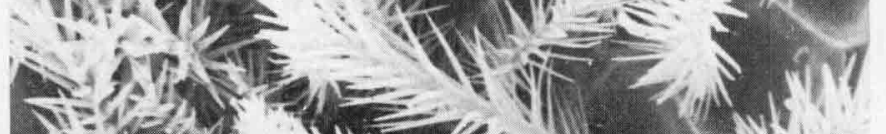
1 1 . Sulve

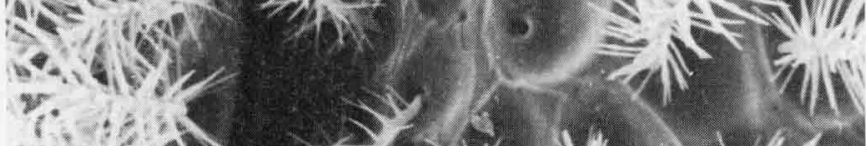

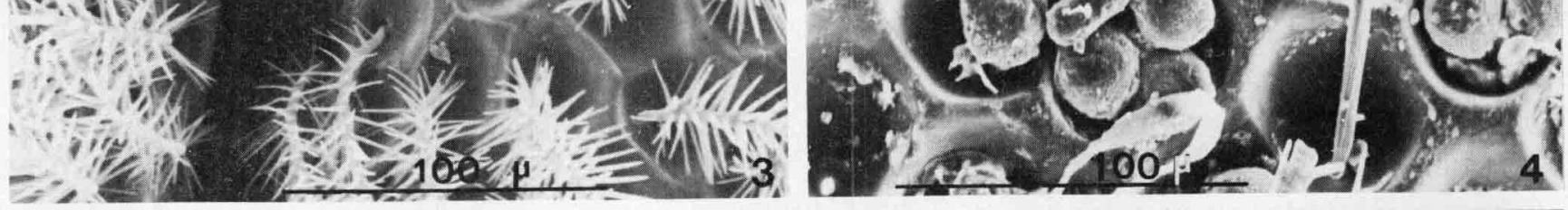

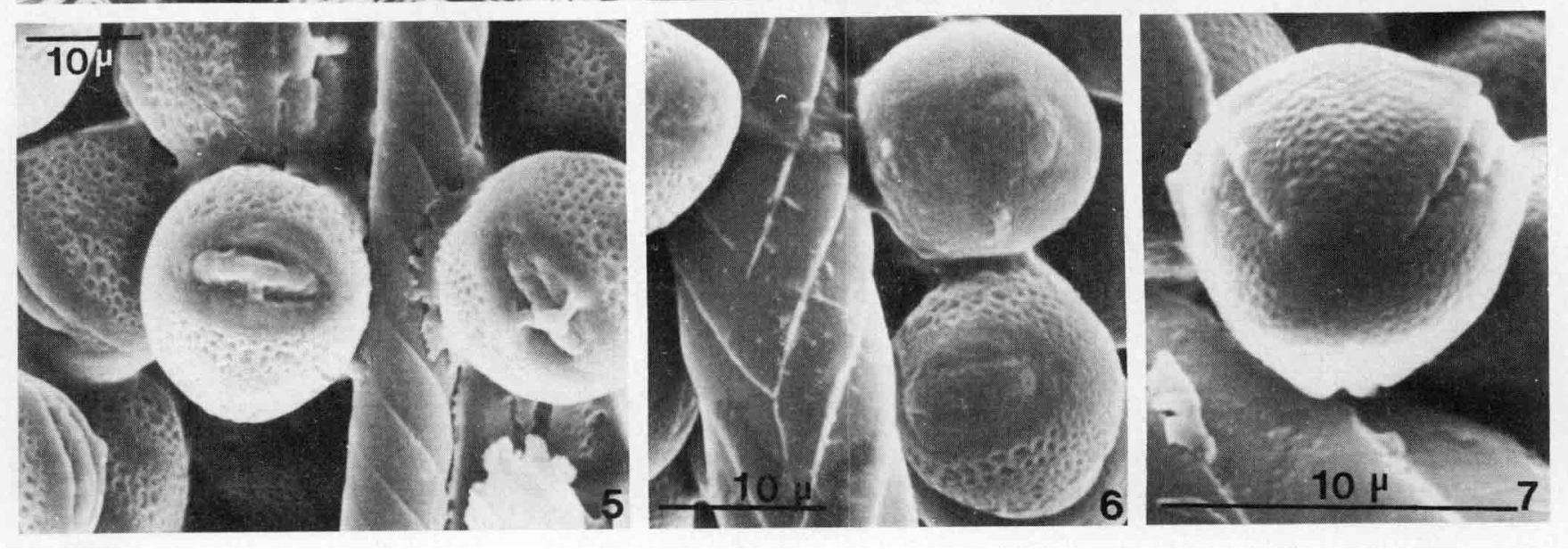


métatarses ou sur le métasternum de l'insecte, malgré des analyses minutieuses portant parfois sur chacune des deux pelotes. Parmi ceux-ci, un seul individu de Xylocopa olivacea capturé sur Sesbania sp. portait des pelotes qui n'avaient pas la même composition : l'une d'entre elles contenait uniquement du pollen de Tephrosia, l'autre 60 p. 100 de grains de Sesbania sp. et 40 p. 100 de Tephrosia sp. (fig. 6). Il semble donc que ces Apoïdes confectionnent, soit les 2 pelotes en même temps : ces dernières ont alors la même composition, ce qui est le cas le plus fréquent ; soit au contraire une première pelote, homogène, puis la seconde, qu'elles complètent éventuellement avec le pollen d'une autre espèce botanique.

Certains individus de Xylocopa capturés en cours de butinage sur des Lamiales : Clerodendron sp., Lantana camara, Ocimum basilicum, n'ont cependant pas collecté de pollen sur ces plantes (LOBREAU-CALLEN \& COUTIN, 1984). Or, la morphologie et la biologie de ces fleurs, toutes tubulaires, sont telles que l'insecte ne peut pas en récolter volontairement le pollen, mais en exploite le nectar. Pour cela elles le dérobent en perforant la base des corolles (FAEGRI \& VAN DER PIJL, 1979). Il est clair, qu'au cours d'un seul voyage, un même individu de xylocope récolte soit du pollen, soit du nectar, soit les deux, selon le type de fleur.

Quel que soit le genre d'Apoïde étudié, plusieurs espèces botaniques ne sont présentes que par une très petite quantité de leurs pollens en proportion inférieure à $0,5 \mathrm{p} .100 \mathrm{du}$ nombre de grains constituant la pelote. Quelle peut être la cause de ce phénomène ? En particulier, l'insecte n'aurait-il pas absorbé préférentiellement ce pollen pour sa propre nourriture ?

\section{Examen comparatif des pollens contenus dans le tube digestif et celui des pelotes}

Chez l'anthophore et la mégachile disséquées, la partie antérieure du tube digestif ne contient aucun pollen dans sa partie thoracique à la différence de la partie postérieure abdominale qui en est largement pourvue. Au contraire, chez les deux individus de Xylocopa olivacea examinés, cette partie antérieure, comme la par- tie postérieure, renfermait, toutes proportions gardées, une grande quantité de pollens.

Dans l'ensemble de ces genres, les pollens extraits des tubes digestifs ont une exine en parfait état de conservation. Seuls les grains les plus gros (Cassia par exemple) sont parfois brisés, déchirés ou perforés par les mandibules lors du «broyage » des étamines. Les exines ne sont pas détériorées par les sucs digestifs qui agissent ainsi sur le contenu des grains à travers les parois. Les exines des pollens renfermés dans les excréments sont pressées et donc déformées, aplaties le plus souvent parallèlement aux apertures.

- Pollens collectés et ingérés par l'anthophore, Amegelia torrida, capturée sur Tephrosia purpurea Pers.

Le contenu digestif est différent de celui des pelotes, tant par les proportions des principaux types polliniques que par le nombre d'espèces botaniques représentées (tabl. 2).

- Pollens collectés par la mégachile, Amegachile geoffreyi, capturée sur Sesbania pachycarpa.

Comme dans le cas précédent, la composition du contenu du tube digestif est plus variée que celle de la pelote portée par la brosse ventrale (tabl. 3).

- Pollens collectés par deux individus de Xylocopa olivacea :

\section{$X$. olivacea capturé sur Helianthus annuus.}

A l'exception d'un individu sur 5 qui portait sur ses pelotes du pollen de Papilionaceae (tabl. 4), les 4 autres capturés en cours de butinage sur $H$. annuus n'avaient pratiquement récolté que du pollen de tournesol, jusqu'à $99 \mathrm{p} .100$.

L'examen du contenu de son tube digestif a conduit à la même constatation. Il semblerait donc que cet individu était fortuitement de passage sur le capitule qu'il n'a pas eu l'opportunité d'exploiter.

\section{$X$. olivacea capturé sur Sesbania pachycarpa.}

La composition pollinique de l'ingestat et celle du contenu de la pelote sont assez comparables (tabl. 5) : il est vraisemblable que le polylectisme des xylocopes est aussi important pour l'alimentation de l'adulte que pour celle de ses larves.

\section{Figure 1}

Soies chevronnées du tarse de la patte postérieure d'un individu de Xylocopa olivacea capturé sur une inflorescence d'Helianthus annuus, Sénégal, Bambey, 24 septembre 1966.

Tarsal herring-bone bristles of the hind-leg of Xylocopa olivacea after foraging on sunflower capitulum.

\section{Figure 2}

Soies à arrêtoir de la corbeille tarsale du même individu, la pelote de pollen étant retirée.

On the same specimen, bristles of corbicula with widen ent. without pollen ball.

\section{Figure 3}

Soies mésonotales plumeuses entières, ne retenant pas le pollen, insérées au niveau d'une suture dorsale du thorax d'un individu femelle de Platysta platystoma capturé sur Sesbania pachycarpa, Sénégal, Bambey, 6 octobre 1969.

Mesotonal whole feather-shape bristles of Platysta platystoma inserted between two sclerites. They are unable to retain pollen strongly.

\section{Figure 4}

Soies mésotonales brisées du même individu. Dans ces conditions le pollen est transporté passivement au fond des cupules d'insertion des soies.

Mesotonal broken bristles of the same specimen of $\mathrm{P}$. platystoma ; pollen grains adhere to the basal cupules around bristle-base.

Figure 5

Pollen de Sesbania sp. sur la brosse ventrale d'un Platysta platystoma.

Sesbania sp. pollen in the same situation.

Figure 6

Pollens de Sesbania sp. et de Tephrosia sp. sur les soies chevronnées de la patte postérieure d'un individu femelle de Xylocopa olivacea. Sesbania and Tephrosia pollen on herring-bone bristles of hind-leg of $\mathrm{X}$. olivacea.

Figure 7

Soies chevronnées de la corbeille de la patte postérieure d'une femelle de Xylocopa olivacea portant du pollen de Crotalaria sp.

Herring-bone bristles of hind-leg corbicula of $\mathrm{X}$. olivacea. 
D. LOBREAU-CALLEN \& R. COUTIN
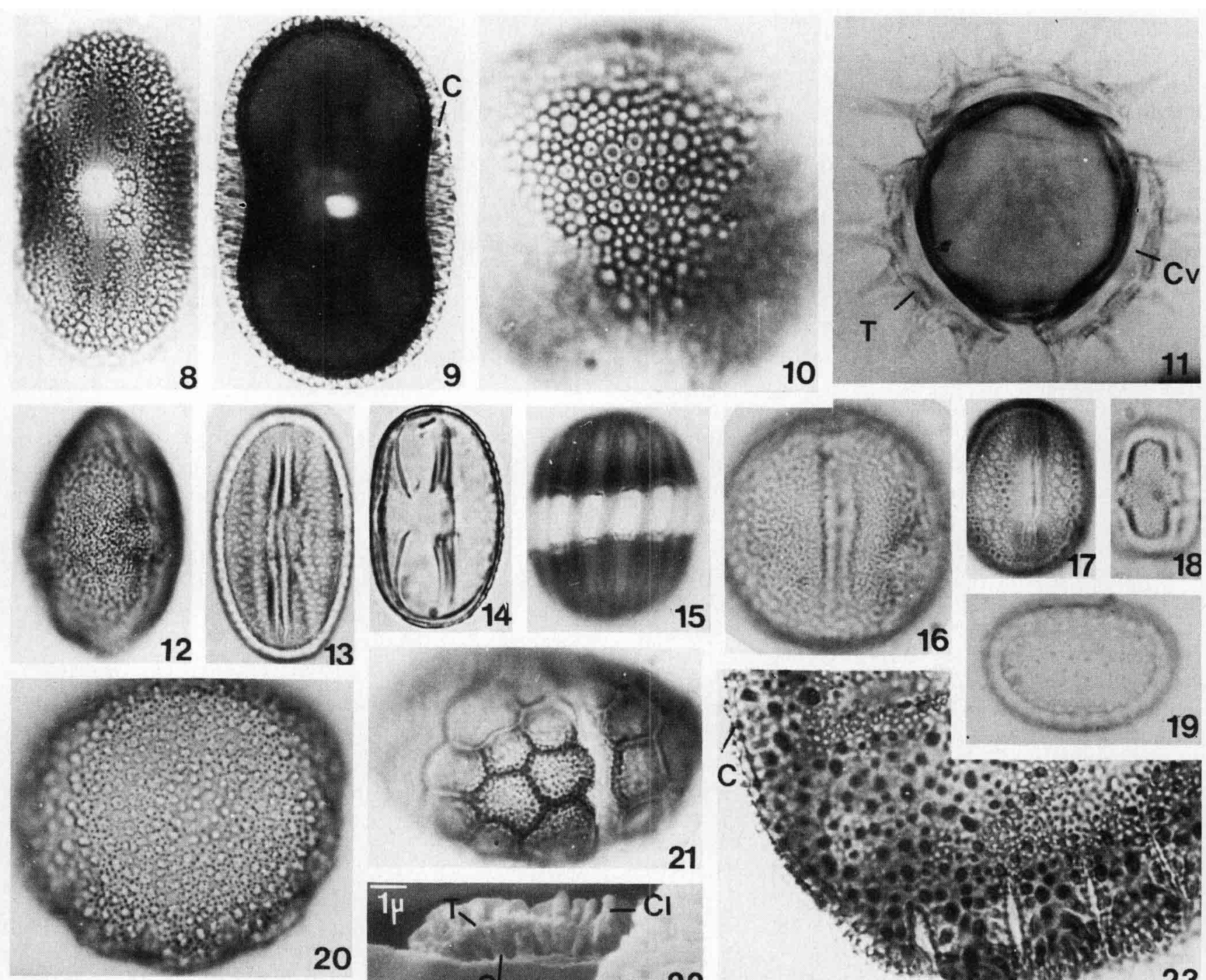

A.

19

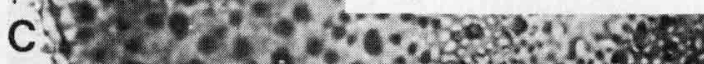

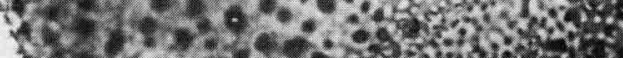

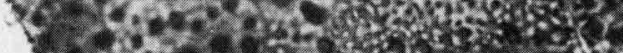

21

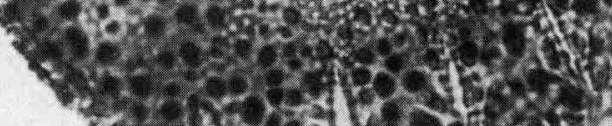

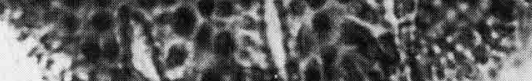
22
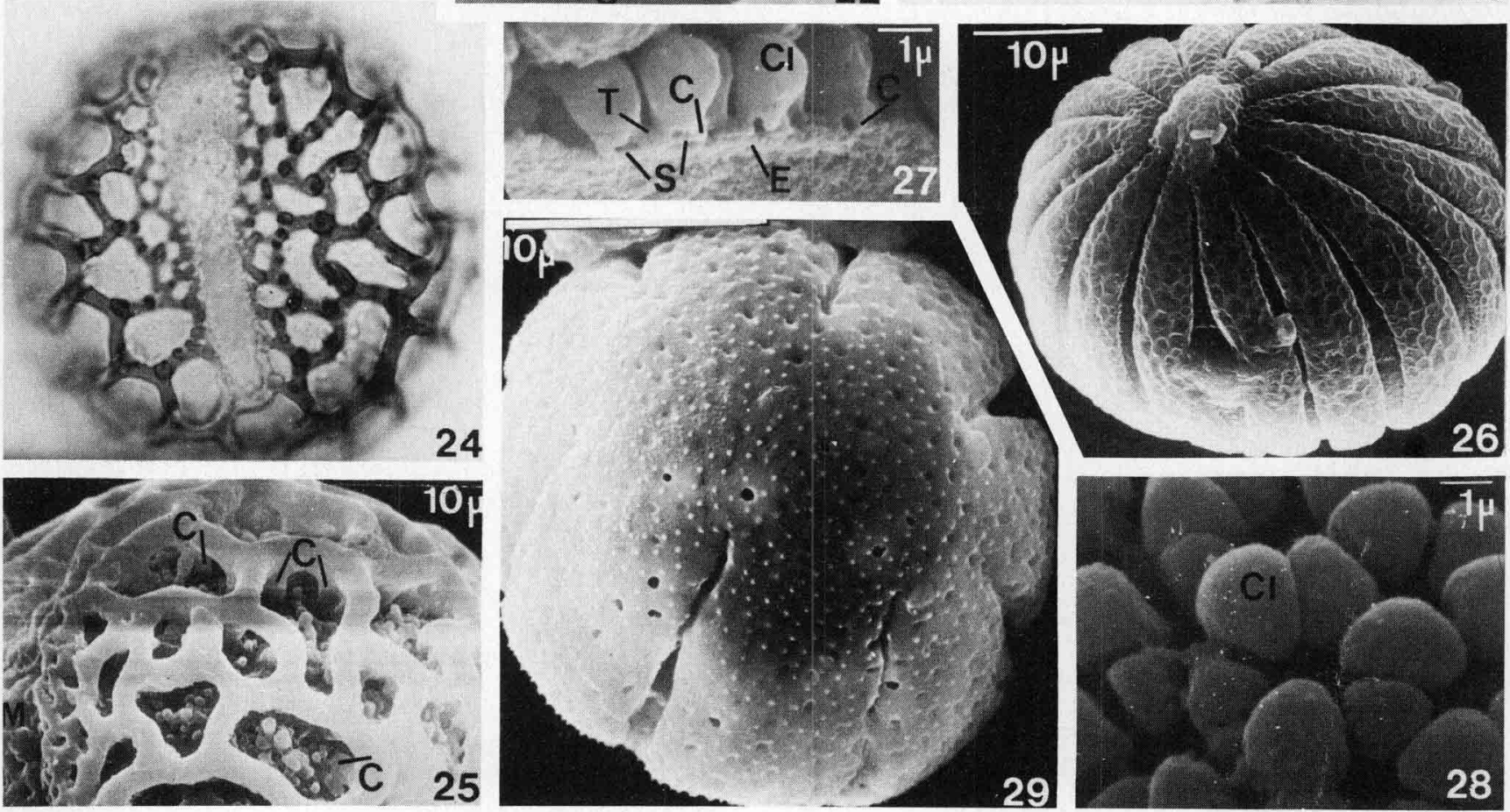

Figures 22,27 et $28-=1 \mu \mathrm{m}$.

Figures 25,26 et $29-=10 \mu \mathrm{m}$ 
Enfin, constatation qui demanderait une étude plus approfondie doublée d'observations comportementales : pourquoi une telle abondance de pollens à l'intérieur de l'intestin antérieur des xylocopes ? S'agit-il de pollens destinés à être régurgités pour la confection de la pâtée pollinique ou non, étant donné que la proportion entre le nombre de grains de pollens des différents taxons est sensiblement plus proche de celle observée sur les pelotes que de celle contenue dans le tractus digestif abdominal.

\section{Caractères floraux des taxons butinés}

L'analyse pollinique des contenus digestifs et des récoltes destinées à l'alimentation des larves montre une certaine différence. La sélection des espèces végétales correspond-elle à plusieurs types floraux ?

Dans le tableau 6 sont résumés les principaux caractères floraux attractifs pour les abeilles dans les espèces ayant fait l'objet de récoltes. Ces caractères sont : la symétrie des inflorescences lorsqu'elles sont denses;
Figures 8 à 29

Morphologie des pollens rencontrés dans les pelotes des Apoïdes solitaires.

Morphology of the pollen grains found in the corbicula of the solitary Apoids.

\section{Figures 8-9}

Justicia striata, apertures, exine tectée perforée ou réticulée.

Justicia striata, apertures, tectate and perforate or reticulate exine.

Figure 10

Hugonia planchoni, vue polaire, exine clavée avec des microépines. Hugonia planchoni, polar view, clavate exine with microechinules.

\section{Figure 11}

Helianthus annuus, pollen d'un individu polyploide, coupe optique équatoriale, tectum structuré avec des columelles, cavae.

Helianthus annuus, pollen of a polyploid specimen, equatorial optical cross, tectum structured with columellae, cavae.

Figure 12

Corchorus olitorius, intercolpium.

Corchorus olitorius, intercolpium.

Figures 13-14

Fagonia cretica, 13, apertures, réseau ; 14, coupe optique méridienne.

Fagonia cretica, 13, apertures, net ; 14, meridional optical cross.

\section{Figure 15}

Polygala sp., apertures nombreuses, toutes centrées sur l'équateur. Polygala $s p .$, numerous apertures, all centred on the equator.

Figure 16

Alysicarpus ovalifolius, sillon et tectum finement verruqueux. Alysicarpus ovalifolius, ectoaperture and finely verrucate tectum.

Figure 17

Sesbania sp., apertures, marge lisse, réseau.

Sesbania sp., apertures, smooth margin, net.

Figure 18

Cf. Ormocarpum sp., intercolpium.

$C f$. Ormocarpum sp., intercolpium.

\section{Figure 19}

Commelina forskalaei, épines tectales alignées en bandes parallèles. Commelina forskalaei, tectale spinules aligned in parallel bands.

\section{Figure 20}

Borreria scaber, vue polaire, tectum perforé et échinulé.

Borreria scaber, polar view, perforate and echinulate tectum.
Figure 21

Ocimum basilicum, pollen colpé, réseau à larges mailles obstruées par un tectum perforé, reposant sur de fines columelles, mur simplicolumellé.

Ocimum basilicum, colpate pollen, net with wide mesh blocked by a perforate tectum layed on fine columellae, simplicolumellate wall.

Figure 22

Sesamum alatum, cassure de l'exine dans un intercolpium, couche infratectale columellaire.

Sesamum alatum, break of the exine in an intercolpium, columellar infratectal layer.

Figure 23

Borreria $c f$. bumbusicola, apertures nombreuses, tectum échinule, perforé, columelles.

Borreria $c f$. bambusicola, apertures, echinulate and perforate tectum, columellae.

\section{Figures 24-25}

Delonix regia, 24, apertures, réseau à larges mailles; 25 , même pollen, membrane aperturale granuleuse, mur simplicolumelle, clavae (cl) dans les mailles.

Delonix regia, 24, apertures, net with broad mesh; 25 , same pollen, granulate apertural membrane, simplicolumellate wall, clavae in the meshes.

\section{Figure 26}

Hygrophila $s p$. sur Xylocopa olivacea, 8 nov. 1968.

Hygrophila $s p$. on Xylocopa olivacea.

\section{Figures 27-28}

Croton sp., cassure de l'exine, clavae sur un mince tectum, columelles courtes, sole lisse, endexine endosculptée $; 28$, structure « crotonoïde " vue au microscope électronique à balayage.

Croton sp., break of the exine, clavae on a thin tectum, short columellae, smooth foot-layer, endosculpturate endexine; 28 , "crotonoid structure" viewed with the SeM.

Figure 29

Mitracarpus sp. sur Amegelia sp., 29 oct. 1968.

Pollen of Mitracarpus $s p$. on Amegelia $s p$.

Figures 1 à 7,26 et 29 , pollens non traités ; Figures 8 à 25, 27, 28, pollens acétolysés.

Figures 1 to 7, 26 and 29, non acetolysed pollen;

Figures 8 to 25, 27, 28, acetolysed pollen.

Les pollens vus au microscope photonique (fig. 8 à $21,23,24)$ sont tous au grossissement 1000.

Pollen grains viewed with the light microscope (fig. 8 to 21, 23, 24) all magnified by 1000 .

Abréviations: $C=$ Columelles; $C V=$ cavae; $C l=$ clava $E=$ endexine $; M=$ membrane aperturale $; S=$ sole $; T=$ tectum. Abbreviations: $C=$ columellae : $C v=$ cavae: $C l=$ clavae; $E=$ endexine $; M=$ apertural membrane; $S=$ foot-layer; $T=$ tectum . 
TABLEAU 2

Proportion entre les pollens des 5 taxons botaniques contenus dans le tube digestif et dans l'une des pelotes d'un individu femelle de l'Anthophoridae, Amegelia torrida qui butinait les fleurs d'un pied de Tephrosia purpurea.

Proportion of pollen from five plant taxa in the hind-intestine and on the corbicula of one hind-leg of Amegelia torrida, after foraging on Tephrosia purpurea flowers.

\begin{tabular}{|c|c|c|c|c|c|}
\hline \multirow{2}{*}{$\begin{array}{l}\text { Taxons botaniques } \\
\text { Botanical taxons }\end{array}$} & \multirow{2}{*}{$\begin{array}{l}\text { Famille } \\
\text { Family }\end{array}$} & \multirow{2}{*}{$\begin{array}{l}\text { Type floral } \\
\text { Floral type }\end{array}$} & \multirow{2}{*}{$\begin{array}{l}\text { Matériel récolté } \\
\text { Harvesting }\end{array}$} & \multicolumn{2}{|c|}{$\begin{array}{l}\text { Pourcentage des pollens récoltés } \\
\text { Per cent of pollen }\end{array}$} \\
\hline & & & & $\begin{array}{l}\text { Tube digestif } \\
\text { Hind-intestine }\end{array}$ & $\begin{array}{l}\text { Pelote } \\
\text { Pollen-ball }\end{array}$ \\
\hline Cassia absus, C. siamea & Caesalpiniaceae & Z & $\mathrm{P}$ & 84,0 & 12,0 \\
\hline Tephrosia purpurea & Papilionaceae & Z & $\mathrm{N}, \mathrm{P}$ & 5,8 & 88,0 \\
\hline Casuarina equisetifolia & Casuarinaceae & A & $\mathrm{P}$ & $\varepsilon$ & \\
\hline Indéterminés & Combretaceae & A & $N, P$ & $\varepsilon$ & \\
\hline
\end{tabular}

$\mathrm{A}=$ actinomorphe $; \mathrm{Z}=$ zygomorphe $; \mathrm{N}=$ nectar $; \mathrm{P}=$ pollen $; \varepsilon$ indique que le pourcentage de pollen de l'espece considerée est inlérieur à $0,3 \%$.

$\mathrm{A}=$ actinomorphic $; \mathrm{Z}=$ zygomorphic $; \mathrm{N}=$ nectar $; \mathbf{P}=$ pollen grains $; \varepsilon \%$ pollen grains less than $0.3 \%$.

TABLEAU 3

Proportion entre les pollens des 3 taxons botaniques contenus dans le tube digestif et sur la brosse ventrale d'un individu femelle de la Megachilidae, Amegelia geoffreyi qui butinait les fleurs d'un pied de Sesbania pachycarpa.

Proportion of pollen from three plant taxa in the hind-intestine and on the ventral brush of an Amegachile geoffreyi female, Sesbania pachycarpa flowers.

\begin{tabular}{|c|c|c|c|c|c|}
\hline \multirow{2}{*}{$\begin{array}{l}\text { Taxons botaniques } \\
\text { Botanical taxons }\end{array}$} & \multirow{2}{*}{$\begin{array}{l}\text { Famille } \\
\text { Family }\end{array}$} & \multirow{2}{*}{$\begin{array}{l}\text { Type floral } \\
\text { Floral type }\end{array}$} & \multirow{2}{*}{$\begin{array}{l}\text { Matériel récolté } \\
\text { Harvesting }\end{array}$} & \multicolumn{2}{|c|}{$\begin{array}{l}\text { Pourcentage des pollens récoltés } \\
\text { Per cent of pollen }\end{array}$} \\
\hline & & & & $\begin{array}{l}\text { Tube digestif } \\
\text { Hind-intestine }\end{array}$ & $\begin{array}{c}\text { Pelote } \\
\text { Pollen-ball }\end{array}$ \\
\hline Sesbania pachycarpa & Papilionaceae & Z & $\mathrm{N}, \mathrm{P}$ & 54,0 & 100 \\
\hline Crotalaria sp. & Papilionaceae & $\mathrm{Z}$ & $\mathrm{N}, \mathrm{P}$ & 45,8 & \\
\hline
\end{tabular}

$\mathrm{A}=$ actinomorphe $; \mathrm{Z}=$ zygomorphe $; \mathrm{N}=$ nectar $; \mathrm{P}=$ pollen $; \varepsilon$ indique que le pourcentage de pollen est inférieur à $0,3 \%$. $\mathrm{A}=$ actinomorphic $; \mathrm{Z}=$ zygomorphic $; \mathrm{N}=$ nectar $; \mathrm{P}=$ pollen grains $; \varepsilon \%$ pollen grains less than $0.3 \%$.

TABLEAU 4

Proportion entre les pollens des 6 taxons botaniques contenus dans le tube digestif et portés par chacune des pattes d'un individu femelle de Xylocopa olivacea qui butinait un capitule d'Helianthus annuus.

Proportion of pollen from six plant taxa in the hind-intestine and on each corbicula of the hind-legs of a Xylocopa olivacea female, after foraging on a Helianthus annuus capitulum.

\begin{tabular}{|c|c|c|c|c|c|c|}
\hline \multirow{3}{*}{$\begin{array}{l}\text { Taxons botaniques } \\
\text { Botanical taxons }\end{array}$} & \multirow{3}{*}{$\begin{array}{l}\text { Famille } \\
\text { Family }\end{array}$} & \multirow{3}{*}{$\begin{array}{l}\text { Type floral } \\
\text { Floral type }\end{array}$} & \multirow{3}{*}{$\begin{array}{l}\text { Matériel récolté } \\
\text { Harvesting }\end{array}$} & \multicolumn{3}{|c|}{$\begin{array}{l}\text { Pourcentage des pollens récoltés } \\
\text { Per cent of pollen }\end{array}$} \\
\hline & & & & \multirow[t]{2}{*}{$\begin{array}{l}\text { Tube digestif } \\
\text { Hind-intestine }\end{array}$} & \multicolumn{2}{|c|}{$\begin{array}{c}\text { Pelote } \\
\text { Pollen-ball }\end{array}$} \\
\hline & & & & & $\mathrm{D}$ & $G$ \\
\hline Sesbania pachycarpa & Papilionaceae & $Z$ & $\mathrm{~N}, \mathrm{P}$ & 55,8 & $\varepsilon$ & 60,0 \\
\hline Tephrosia purpurea & Papilionaceae & Z & $\mathrm{N}, \mathrm{P}$ & 43,3 & 99,9 & 40,0 \\
\hline Alchornea sp. & Euphorbiaceae & A & $\mathrm{N}, \mathrm{P}$ & $\varepsilon$ & & \\
\hline Cassia absus, $C$. siamea & Papilionaceae & Z & $\mathrm{P}$ & 0,6 & & \\
\hline Crotalaria sp. & Papilionaceae & Z & $\mathrm{N}, \mathrm{P}$ & & $\varepsilon$ & \\
\hline Pavonia hirsuta & Malvaceae & A & $\mathrm{N}, \mathrm{P}$ & & $\varepsilon$ & \\
\hline
\end{tabular}

$\mathrm{A}=$ actinomorphe $; \mathrm{Z}=$ zygomorphe $; \mathrm{N}=$ nectar $; \mathrm{P}=$ pollen $; \varepsilon$ indique que le pourcentage de pollen est inférieur à $0,3 \%$.

$\mathrm{D}=$ droite $; \mathrm{G}=$ gauche.

$\mathrm{A}=$ actinomorphic $; \mathrm{Z}=$ zygomorphic $; \mathrm{N}=$ nectar $; \mathrm{P}=$ pollen $; \varepsilon \%$ pollen grains less than $0.3 \%$.

$\mathrm{D}=$ right leg $; \mathrm{G}=$ left leg. 
TABLEAU 5

Proportion entre les pollens des 7 taxons botaniques contenus dans le tube digestif et ceux de l'une des pelotes d'un individu femelle de Xylocopa olivacea capturé sur les fleurs d'un pied de Sesbania pachycarpa.

Proportion of pollen from seven plant taxa in the hind-intestine and on the corbicula of one hind-leg of a Xylocopa olivacea female after foraging on Sesbania pachycarpa flowers.

\begin{tabular}{|c|c|c|c|c|c|}
\hline \multirow{2}{*}{$\begin{array}{l}\text { Taxons botaniques } \\
\text { Botanical taxons }\end{array}$} & \multirow{2}{*}{$\begin{array}{l}\text { Famille } \\
\text { Family }\end{array}$} & \multirow{2}{*}{$\begin{array}{l}\text { Type floral } \\
\text { Floral type }\end{array}$} & \multirow{2}{*}{$\begin{array}{l}\text { Matériel récolté } \\
\text { Harvesting }\end{array}$} & \multicolumn{2}{|c|}{$\begin{array}{c}\text { Pourcentage des pollens récoltés } \\
\text { Per cent of pollen }\end{array}$} \\
\hline & & & & $\begin{array}{l}\text { Tube digestif } \\
\text { Hind-intestine }\end{array}$ & $\begin{array}{c}\text { Pelote } \\
\text { Pollen-ball }\end{array}$ \\
\hline Cassia absus, Cassia siamea & Caesalpiniaceae & $\mathrm{Z}$ & $\mathbf{P}$ & 91,7 & 81,6 \\
\hline Justicia striata & Acanthaceae & $\mathrm{Z}$ & $\mathrm{N}, \mathrm{P}$ & 6,9 & 15,3 \\
\hline Sesbania pachycarpa & Papilionaceae & $\mathrm{Z}$ & $\mathrm{N}, \mathrm{P}$ & 0,4 & 1,2 \\
\hline Vigna unguiculata & Papilionaceae & $\mathrm{Z}$ & $\mathrm{N}, \mathrm{P}$ & 0,7 & \\
\hline Cucumis sp. & Cucurbitaceae & $\mathrm{Z}$ & $\mathrm{N}, \mathrm{P}$ & $\varepsilon$ & 0,4 \\
\hline Commelina forskalaei & Commelinaceae & $\mathrm{Z}$ & $\mathrm{N}, \mathrm{P}$ & & $\varepsilon$ \\
\hline Borreria sp. & Rubiaceae & $\mathrm{Z}$ & $\mathbf{P}$ & & $\varepsilon$ \\
\hline Indéterminés & & & $\mathbf{P}$ & & $\varepsilon$ \\
\hline
\end{tabular}

$\mathrm{A}=$ actinomorphe $; \mathrm{Z}=$ zygomorphe $; \mathrm{N}=$ nectar $; \mathrm{P}=$ pollen $; \varepsilon$ indique que le pourcentage de pollen est inférieur à $0,3 \%$. $\mathrm{A}=$ actinomorphic $; \mathrm{Z}=$ zygomorphic $; \mathrm{N}=$ nectar $; \mathrm{P}=$ pollen $; \varepsilon \%$ pollen grains of the considered species less than $0.3 \%$.

les abeilles ne voyant que l'ensemble, ou celle des fleurs lorsqu'elles sont isolées ou groupées en inflorescences lâches ; la couleur des pétales et leurs soudures ; la présence de nectar et leurs organes sécréteurs ; la déhiscence des étamines qui conditionne la technique d'extraction des pollens par vibrations en cas de déhiscence poricide.

Dans le tableau 6, les familles sont arrangées selon la classification naturelle des Angiospermes dans le but de regrouper celles dont certains caractères morphologiques sont apparentés.

D'après l'inventaire des espèces butinées par les différents genres d'Apoïdes étudiés, il apparaît ainsi que les Anthophora recherchent pour leur alimentation, des fleurs groupées en inflorescences denses, dialypétales, de teinte claire et avec de nombreuses étamines produisant ainsi une grande quantité de pollens. En revanche, les fleurs choisies pour les récoltes destinées aux larves sont plus ou moins zygomorphes, gamopétales et tubulaires ; elles produisent un nectar concentré soit riche en hexoses (Helianthus, Centaurea...) ou en saccharose (Ocimum), soit qui possède tous les types de sucres en proportions variables, selon le lieu de végétation, les variations climatiques et hygrométriques, les heures de la journée (WILLMER, 1980 ; SOUTHWICK et al., 1981 ; BAKER \& BAKER, 1983 ; FRANKIE \& HABER, 1983). D'après ces données, les anthophores ne semblent pas récolter particulièrement tel ou tel type de sucre, si ce n'est en fonction de leur concentration dans le nectar. Les espèces butinées pour la nutrition du couvain appartiennent presque toutes à la sous-classe évoluée des Asteridae.

Dans le cas des Megachile, qu'il s'agisse de leur alimentation propre ou de celle de leurs larves, elles recherchent exclusivement les fleurs dialypétales zygomorphes des Papilionaceae (Rosidae, sous classe moyennement évoluée) dont l'étendard est dressé, de teintes claires et vives. Il semble par ailleurs que le nectar, comme le pollen de cette sous-famille soit indispensable à la maturation des ovocytes et à leur fécondité optimale. D'ailleurs l'appétance des mégachiles pour les Papilionacae est bien connue (TASEI, 1977).

En ce qui concerne les $X y$ locopa, lorsqu'ils récoltent le pollen, tant pour leur alimentation que pour celle des larves, ils montrent une attractivité particulière pour les fleurs dialypétales zygomorphes de teintes claires et vives des Légumineuses (Rosidae), ainsi que pour les fleurs groupées en grosses inflorescences très denses (Asteridae). Par contre, lorsqu'ils récoltent le nectar, ils ne marquent aucune préférence marquée pour les fleurs dialy/ ou gamopétales.

Il est ainsi possible de conclure qu'il existe des relations coadaptatives entre ces Apoïdes et certains groupes botaniques relativement évolués (Rosidae et Asteridae) lorsqu'il s'agit de l'alimentation des larves. Par opposition, il n'existe aucun lien insectes/plantes lorsqu'il s'agit de l'alimentation des adultes.

- Caractères morphologiques des pollens récoltés par les Apoïdes

Les pollens récoltés par ces Apoïdes solitaires ont en commun la structure de l'exine : les pollens sont tectés et le tectum qui porte l'ornementation est lisse (fig. 5) et perforé (fig. $7,16,23$ ) ou parfois aussi aréolé (fig. 8), clavé (fig. 10, 22, 27, 28), échinulé (fig. 11, $19,20,29)$; dans quelques cas les grains sont réticulés avec des mailles soit de grand diamètre et obstruées par un tectum perforé (fig. 12, 22, 26), soit de petit diamètre, séparées par un mur pouvant être de la même largeur que le diamètre des mailles (fig. 13, 17, 18).

Cette structure est telle que le tectum occupe, dans tous les cas, au moins 50 p. 100 de la surface du pollen. En outre, ce tectum est massif (fig. 22, 27) et n'est structuré avec des columelles que chez les Asteraceae où la couche infratectale est fréquemment remplacée par des cavae (fig. 11). Seul le pollen de Delonix regia (Boj.) Raf. fait exception avec son exine largement réticulée (fig. 24, 25). La fréquence de ces grains est cependant très faible et peut s'expliquer par le fait que les oiseaux et les papillons sont les principaux pollinisateurs de ces fleurs qui sont d'ailleurs de couleur rouge (KALIN ARROYO, 1981).

Par la structure de leur exine, les pollens récoltés par ces Apoïdes ne peuvent avoir que des variations harmomégathiques limitées: les apertures sont peu saillantes lorsque les grains sont turgescents et les 
modifications de volume des pollens sont relativement réduites en cas de dessication. Dans le pollen frais d'Hygrophila senegalensis, l'exine, rigide aux pôles, est régulièrement interrompue de part et d'autre des apertures complexes par des sillons qui répartissent ainsi, dans la région équatoriale du pollen, les forces de tension de l'exine sur toute la surface du grain (fig. 26).

L'exine des pollens de fleurs zoophiles porte également le «pollen-coat» (sensu ZANDONELLA et al., 1981), riche en protéines, lipoïdes et lipides qui renferment des substances volatiles. Il permet la reconnaissance du pollen par les stigmates, le fixe sur le pollinisateur. Cette substance semble également attractive pour certains Apoïdes solitaires (DOBSON, 1984, BUCH-
MAN, 1983, SCHMIDT, 1985). Pour les pollens récoltés par les Apoïdes étudiés ci-dessus, le « pollen-coat » est largement réparti à la surface des grains, les espaces intercolumellaires étant particulièrement réduits (fig. 9, $14,22,23,27)$, même chez les Asteraceae où les cavae en sont dépourvues (HESSE, 1981, FRAGLOWSKI \& GRAFSTROEM, 1980).

\section{RELATIONS ENTRE LES APOÏDES \\ ET LA VÉGÉTATION. LEUR COMPORTEMENT VIS-À-VIS DE LA VÉGÉTATION}

La connaissance des types morphologiques floraux, résumés dans le tableau 6 , et des types polliniques

\section{TABLEAU 6}

Caractères botaniques des taxons reconnus lors des analyses polliniques des pelotes des Apö̈des. Les taxons botaniques sont ordonnés selon la classification phylétique de CRONQUIST (1968): tabl. a, les Asteridae, b, les Rosidae, c, les autres Angiospermes.

Botanical characters of taxa obtained by pollen analysis of pollen balls from solitary bees.

Botanical taxa arranged according to CRONQUIST's phyletic classification (1968) : tabl. 6a, Asteridae, b. Rosidae, $c$, other Angiosperms.

TABLEAU $6 \mathrm{a}$

Les Asteridae.

\begin{tabular}{|c|c|c|c|c|c|c|c|c|}
\hline $\begin{array}{l}\text { Taxons récoltés } \\
\text { par les Apoïdes } \\
\text { Taxa collected } \\
\text { by the Apoids }\end{array}$ & 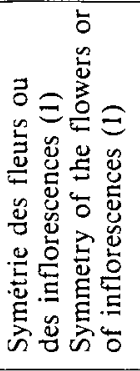 & 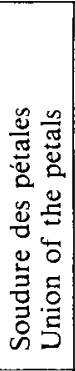 & 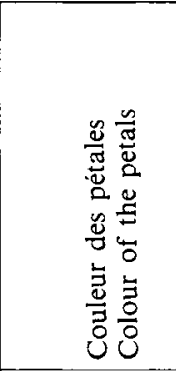 & 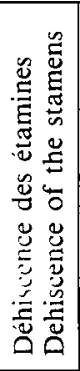 & 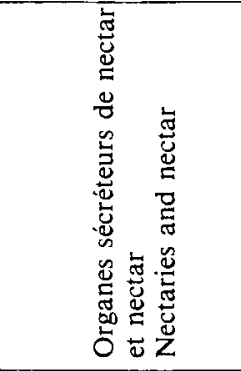 & 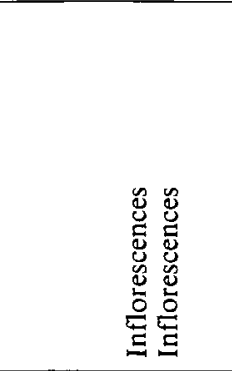 & 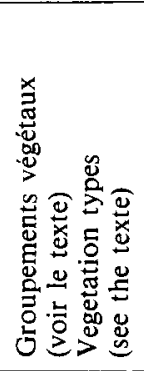 & 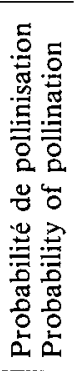 \\
\hline \multicolumn{9}{|l|}{ ASTERIDAE } \\
\hline $\begin{array}{l}\text { Asteraceae } \\
\quad \text { Centaurea senegalensis }\end{array}$ & A & G & Mauve-blanc & $\mathrm{L}$ & $\begin{array}{l}\text { Nectaires à la } \\
\text { base de la corolle }\end{array}$ & Capitule & & + \\
\hline $\begin{array}{l}\text { Helianthus annuus } \\
\text { Rubiaceae }\end{array}$ & A & $\mathrm{G}$ & Jaune & $\mathrm{L}$ & - & 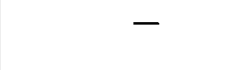 & & + \\
\hline Spermacoce/Borreria & A & G & Blanc & $\mathbf{L}$ & Disque & Glomérule & & + \\
\hline Kauhotia grandiflora & A & G & Rose & $\mathrm{L}$ & - & Corymbe & & \\
\hline $\begin{array}{l}\text { Mitracarpus villosus } \\
\text { Pedaliaceae }\end{array}$ & A & $\mathrm{G}$ & Blanc & $\mathbf{L}$ & - & Glomérule & & \\
\hline $\begin{array}{l}\text { Sesamum alatum, } \\
\text { S. indicum }\end{array}$ & $Z$ & G & Mauve-blanc & $\mathrm{L}$ & $\begin{array}{l}\text { Disque staminal et } \\
\text { poils à mucilage }\end{array}$ & $\begin{array}{l}\text { Fleur solitaire sur } \\
\text { un épi }\end{array}$ & (1) & \\
\hline $\begin{array}{l}\text { Acanthaceae } \\
\text { Hygrophila senegalensis }\end{array}$ & Z & $\mathrm{G}$ & Violacée & $\mathrm{L}$ & Disque & $\begin{array}{l}\text { Glomérule } \\
\text { pauciflore }\end{array}$ & mares & + \\
\hline $\begin{array}{l}\text { Justicia striata } \\
\text { Lamiaceae }\end{array}$ & Z & G & Pourpre & $\mathbf{L}$ & Disque & Epi pauciflore & (1), (5) & + \\
\hline $\begin{array}{l}\text { Ocimum basilicum } \\
\text { Verbenaceae }\end{array}$ & $\mathrm{Z}$ & $\mathrm{G}$ & Blanc & $\mathrm{L}$ & Nectaires, odeurs & Epi pauciflore & & + \\
\hline Lantana camara & Z & G & $\begin{array}{l}\text { Jaune puis } \\
\text { rouge }\end{array}$ & $\mathrm{L}$ & $\begin{array}{l}\text { Nectar et odeurs } \\
\text { Plus de nectar } \\
\text { plus d'odeurs }\end{array}$ & Corymbe & (2), (1) & $?$ \\
\hline $\begin{array}{l}\text { Convolvulaceae } \\
\text { Ipomoea spp. dont } \\
\text { I. batatas }\end{array}$ & A & G & Mauve & $\mathrm{L}$ & Disque staminal & Glomérule & (1) & + \\
\hline $\begin{array}{l}\text { Apocynaceae } \\
\text { Thevetia neriifolia }\end{array}$ & A & G & Jaune & $\mathrm{L}$ & Nectaires, odeurs & Fleurs isolées & (1) & + \\
\hline
\end{tabular}

Légende commune aux tableaux $6 \mathrm{a}, \mathrm{b}, \mathrm{c}$ :

$\mathrm{A}=$ actinomorphe $; \mathrm{Z}=$ zygomorphe $; \mathrm{G}=$ gamopétale $; \mathrm{D}=$ dialypétale $; \mathrm{L}=$ déhiscence longitudinale $; \mathrm{P}=$ déhiscence poricide.

(1) Symétrie des fleurs ou des inflorescences denses telle qu'elle apparaît aux insectes.

Les chiffres 1 à 5 définissant des groupements végétaux sont expliqués dans le texte.

Legend common to the tables $2 \mathrm{a}, \mathrm{b}, \mathrm{c}$ :

$\mathrm{A}=$ actinomorphic $; \mathrm{Z}=$ zygomorphic $; \mathrm{G}=$ gamopetalous $; \mathrm{D}=$ dialypetalous $; \mathrm{L}=$ longitudinal dehiscence $; \mathrm{P}=$ dehiscence by pore.

(1) Symmetry of the flowers or of the dense inflorescences as the Apoids see them.

The numbers 1 to 5 defining the vegetation types are explained in the text. 
TABLEAU $6 b$

Les Rosidae.

\begin{tabular}{|c|c|c|c|c|c|c|c|c|}
\hline $\begin{array}{l}\text { Taxons récoltés } \\
\text { par les Apoides } \\
\text { Taxa collected } \\
\text { by the Apoids }\end{array}$ & 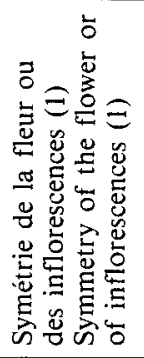 & 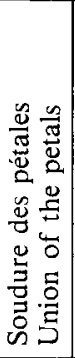 & 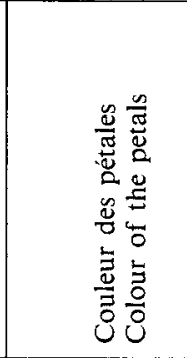 & 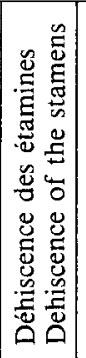 & 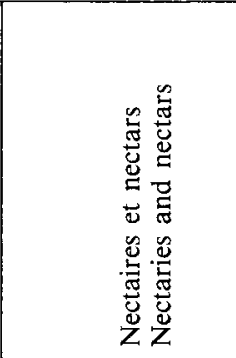 & 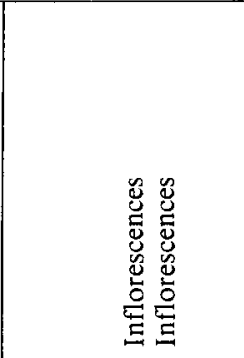 & 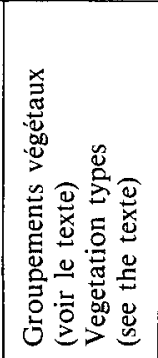 & 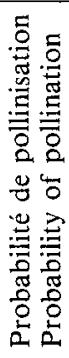 \\
\hline \multicolumn{9}{|l|}{ ROSIDAE } \\
\hline $\begin{array}{l}\text { Polygalaceae } \\
\text { Polygala sp. } \\
\text { Linaceae }\end{array}$ & $\mathrm{Z}$ & G & & $P$ & Disque staminal & Fleurs isolées & (1), (2), (4) & + \\
\hline $\begin{array}{l}\text { Hugonia planchonii } \\
\text { Zygophyllaceae }\end{array}$ & A & $\mathrm{D}$ & Jaune & $\mathrm{L}$ & Disque staminal & Cyme & (1) & + \\
\hline $\begin{array}{l}\text { Fagonia cretica } \\
\text { Leguminosae }\end{array}$ & A & D & Rose & $\mathrm{L}$ & disque & Fleurs isolées & (1), (4), (5) & + \\
\hline $\begin{array}{c}\text { Papilionaceae } \\
\text { Alysicarpus ovalifolius }\end{array}$ & $\mathrm{Z}$ & D & Pourpre & $\mathrm{L}$ & $\begin{array}{l}\text { Calice glanduleux } \\
\text { discifère }\end{array}$ & Grappe & (1) & + \\
\hline Crotalaria spp. & $\mathrm{z}$ & D & Jaune & $\mathrm{L}$ & - & - & (1), (5) & + \\
\hline Indigofera st & $\mathrm{Z}$ & D & Rose-pourpre & $\mathrm{L}$ & - & Epi & (1), (2), (5) & + \\
\hline & $\mathrm{Z}$ & D & Rose, $\mathrm{b}$ & $\mathrm{L}$ & - & Grappe & (1), & \\
\hline & $\mathrm{z}$ & D & Jaun & $\mathrm{L}$ & - & - & (1) & + \\
\hline Tephrosia purpurea & $\mathrm{Z}$ & D & Rose, rouge & $\bar{L}$ & - & - & (1), (5) & + \\
\hline Vigna unguiculata & $\mathrm{Z}$ & D & Bleu, jaune & L & - & Epi & (1) & + \\
\hline $\begin{array}{c}\text { Caesalpiniaceae } \\
\text { Caesalpinia pulcherrima }\end{array}$ & $\mathrm{Z}$ & D & Rouge, jaune & $\mathrm{L}$ & Calice glanduleux & Grappe & (1) & 0 \\
\hline Cassia siamea, C. absus & $\mathrm{Z}$ & $\mathrm{D}$ & Jaune & L & Staminodes & Grappe & (1), (2) & \\
\hline Delonix regia & $\mathrm{Z}$ & D & Rouge & $\mathrm{L}$ & Calice glanduleux & Grappe & 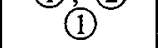 & 0 \\
\hline Mimosaceae & & & & & & & & \\
\hline Acacia ataxacantha & $\mathrm{Z}$ & $\mathrm{L}$ & Blanchâtre & L & Calice glanduleux & Epi de glomérules & (4), (5) & + \\
\hline
\end{tabular}

TABLEAU $6 \mathrm{c}$

Les Dicotylédones : les Dilleniidae et les Caryophylliidae, et les Monocotylédones.

\begin{tabular}{|c|c|c|c|c|c|c|c|c|}
\hline $\begin{array}{l}\text { Taxons récoltés } \\
\text { par les Apoïdes } \\
\text { Taxa collected } \\
\text { by the Apoids }\end{array}$ & 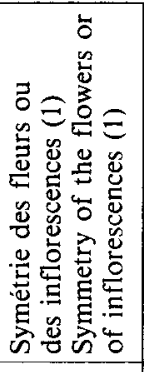 & 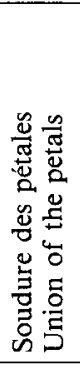 & 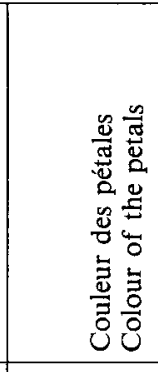 & 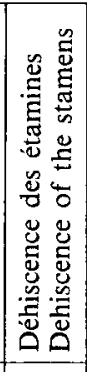 & 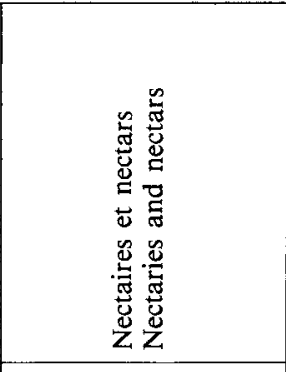 & 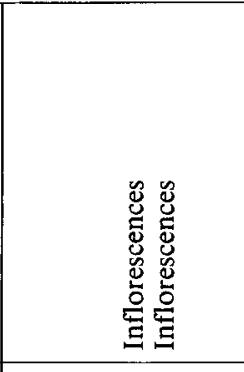 & 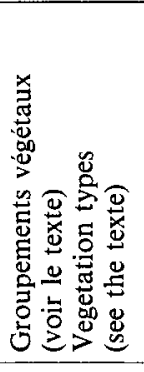 & 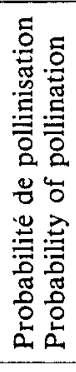 \\
\hline \multicolumn{9}{|l|}{ DILLENIIDEAE } \\
\hline $\begin{array}{l}\text { Cucurbitaceae } \\
\text { Luffa sp. } \\
\text { Cucumis sp. }\end{array}$ & $\begin{array}{l}\text { A } \\
\text { A }\end{array}$ & $\begin{array}{l}\mathrm{G} \\
\mathrm{G}\end{array}$ & \begin{tabular}{|l} 
Jaune \\
Jaune
\end{tabular} & $\begin{array}{l}\mathbf{L} \\
\mathbf{L}\end{array}$ & $\begin{array}{c}\text { Nectaires corolliflores } \\
-\end{array}$ & Fleurs isolées & (1) & $\begin{array}{l}+ \\
+\end{array}$ \\
\hline $\begin{array}{l}\text { Malvaceae } \\
\text { Pavonia hirsuta }\end{array}$ & A & D & Jaune & $\mathrm{L}$ & $\begin{array}{l}\text { Nectaires à la } \\
\text { base du calice }\end{array}$ & Grappe pauciflore & (1) & + \\
\hline $\begin{array}{l}\text { Tiliaceae } \\
\text { Grewia cf. flavescens }\end{array}$ & A & $\mathrm{D}$ & Jaune & $\mathrm{L}$ & $\begin{array}{l}\text { Nectaires sur } \\
\text { les pétales }\end{array}$ & Glomérule & (1) & + \\
\hline $\begin{array}{l}\text { Corchorus olitorius } \\
\text { CARYOPHYLLIIDAE }\end{array}$ & A & D & - & $\mathbf{L}$ & - & - & (1) & + \\
\hline $\begin{array}{l}\text { Amaranthaceae } \\
\text { Portulacaceae }\end{array}$ & A & D & Verdâtre & L & & Epi & (3) & + \\
\hline $\begin{array}{l}\text { Portulaca } \text { sp. } \\
\text { MONOCOTYLÉDONES }\end{array}$ & A & D & Jaune & $\mathbf{L}$ & & Fleurs isolées & (3) & + \\
\hline $\begin{array}{l}\text { Liliaceae } \\
\text { Cf. Aloe sp. } \\
\text { Poaceae } \\
\text { Commelinaceae }\end{array}$ & $\begin{array}{l}\mathrm{A} \\
\mathrm{A}\end{array}$ & $\begin{array}{l}\text { D } \\
\text { D }\end{array}$ & & $\begin{array}{l}\mathrm{L} \\
\mathrm{L}\end{array}$ & & $\begin{array}{l}\text { Grappe } \\
\text { Epi }\end{array}$ & (4), (5) & $\begin{array}{l}+ \\
0\end{array}$ \\
\hline Commelina forskalaei & $\mathrm{z}$ & $\mathrm{D}$ & Bleu & $\mathbf{L}$ & Staminodes & Grappe/Glomérule & (2) & + \\
\hline
\end{tabular}


sélectionnés pour les récoltes en pollen et en nectar peut permettre de mieux comprendre le rôle éventuel des Apoïdes dans la dynamique de la végétation.

\section{A. Comportement des Apoïdes vis-à-vis de la flore pour la construction des nids et pour les dortoirs}

La fin de la saison des pluies est la période où ces insectes adultes sont particulièrement nombreux. A cette époque, les femelles butinent très activement pour approvisionner leurs nids situés dans des cellules construites dans le sol, tapissées d'une substance sécrétée par l'insecte (Anthophora) ou construites à partir de fragments de feuilles (Megachile) ou encore dans les tiges mortes et creuses d'arbustes comme celles du Nerium oleander L. (Xylocopa). Dans ce dernier cas, les femelles capturées ne portaient aucune trace de pollen de cette espèce.

A Bambey, DESMIER de CHENON (Comm. pers.) a noté que les mégachiles dont les mâles se regroupent en dortoir sur des Graminées telles que Brachiaria distichophylla (Trin.) Stapf et Panicum callocarpum Berh., découpent des feuilles de Waltheria indica L., et de Guiera senegalensis J. F. Gmel. gayanus Kunth. L'analyse pollinique des récoltes destinées à la nourriture des larves ne permet de retrouver aucune trace de ces plantes. Ceci démontre bien l'indépendance complète des différentes séquences d'activité de ces insectes : nourriture de l'individu, construction des cellules, récoltes des réserves nutritives des larves.

\section{B. Comportement des Apoïdes vis-à-vis de la flore pour l'approvisionnement des nids et pour l'ali- mentation. Rôle dans la pollinisation}

Les sites d'où proviennent les insectes capturés appartiennent tous aux régions soudaniennes dont le couvert végétal correspond à 5 groupements qui s'interpénètrent :

- (1), les cultures de la Station de Recherches agronomiques de Bambey (700 ha) qui comprennent sous couvert de la forêt clairsemée à Acacia albida Del. de nombreuses plantes exotiques introduites de longue date, accompagnées de la flore des adventices (MERLIER \& MONTEGUT, 1982) ;

- (2), les haies et les plantes en bordure de champs où se rencontrent à la fois des plantes autochtones et des allochtones souvent cosmopolites (Clitoria ternatea L., Commelina forskalaei Vahl., C. benghalensis L., Centaurea perrottetti DC, Ageratum conyzoides L., ...) ;

- (3), les rudérales nitrophiles (Boerhaavia erecta L., Mitracarpus villosus (Sw.) DC, Merremia aegyptiaca (L.) Urb., Physalis angulata L., ...) ;

- (4), les végétaux autochtones de jachères, souvent ligneux, avec de nombreux sous-arbrisseaux ( Cassia mimosoides L., Hibiscus asper Hook., Heliotropium bacciferum Forsk., Maytenus senegalensis (Lam.) Exell, Maerua sp., Vernonia sp., Zizyphus sp., ...) ;

- (5), les végétaux autochtones de terrains incultes sur sols pauvres, peu profonds, latéritiques ou ayant colonisé des zones de cultures abandonnées depuis longtemps (Guiera senegalensis J. F. Gmel., Combretum micranthum G. Don., Chrozophora senegalensis (Lam.) A. Juss., Tephrosia purpurea Pers., ...).

En général, dans la région étudiée, les plantes cultivées occupent une plus grande surface que les végétaux spontanés. Les éléments fort variés de cette flore étant fleuris à la même période, il existe donc une certaine compétitivité entre eux pour le butinage et leur pollinisation.

Les groupements végétaux auxquels appartiennent les espèces sélectionnées par les insectes pour le butinage sont indiqués dans le tableau 6. A la lecture de ce dernier, il est évident que les groupements 4 et 5 sont les moins fréquentés par les Apoïdes.

Le comportement des Apoïdes peut alors se caractériser de la façon suivante :

- ils ont une réelle préférence, en particulier les mégachiles, pour les espèces de la flore locale lorsque celles-ci participent à des floraisons abondantes, négligeant alors les plantes introduites, surtout les ornementales dont les fleurs de grandes dimensions sont principalement adaptées à la pollinisation par des papillons, des oiseaux ou des chauve-souris (tabl. 6) ;

- elles négligent pratiquement les groupements végétaux naturels, lorsque les inflorescences sont peu odorantes et peu visibles, et sur lesquelles elles entrent peut-être en compétition avec d'autres insectes plus adaptés à cette flore (tabl. 6) ;

- certains Apoïdes, et ce fut souvent le cas des xylocopes, malgré l'abondance des fleurs disponibles, négligent la flore spontanée lorsqu'ils trouvent sur les plantes introduites, cultivées ou non, une source très importante de pollen et de nectar.

Dans cette région de Bambey, parmi les quelques arbres de grandes dimensions, seuls Bombax sp., Khaya senegalensis (Desr.) A. Juss. et Combretum sp. sont fleuris à l'époque considérée. Or, ces essences n'ont pratiquement pas fait l'objet de récoltes de pollen de la part des Xylocopa et des Anthophora qui, comme d'ailleurs les Megachile, semblent ne récolter du pollen que sur la strate végétale herbacée la plus fleurie. Toutefois, certaines Anthophora ont tenté de s'y alimenter.

D'après la liste des plantes mellifères de CRANE et al. (1984), et par l'analyse des pelotes de xylocopes capturés en mai dans ces mêmes régions, les fleurs de ces 2 arbres, Bombax et Combretum, sont pourtant recherchées par les abeilles sociales et solitaires. Or durant la période de la fin de la saison des pluies, seuls les Cetoniinae, en particulier Pachnoda aurantia Hbst., butinent sur ces arbres, surtout sur les Combretum.

Par ailleurs, la présence de plusieurs espèces de grands Buprestidae sur les Légumineuses (Julodis aequinoctialis Ol. sur Sesbania spp. ; Sternocera interrupta Ol. et $S$. castanea Ol. sur Acacia seyal Del.), ne semble pas perturber le butinage de ces plantes par les Apoïdes.

Il semblerait donc que la présence des Cetoniinae sur ces arbres, par opposition à celle des grands Buprest $i$ dae sur d'autres taxons, présentent un caractère répulsif pour les abeilles solitaires. Il existerait donc là en quelque sorte, une certaine compétition entre les Apoïdes et les Cetoniinae. Par observation directe, ce phé- 
nomène de répulsion et de compétition semble reposer sur des caractères d'odeurs et de phéromones, les floraisons n'étant pas attractives pour les Apoïdes...

\section{Rôle des Apoïdes dans la dynamique de la végé- tation}

Les abeilles solitaires de savanes d'autres régions du monde ont-elles un comportement semblable vis-à-vis de la flore de ces pays?

Il semble que oui, car au Costa Rica, en zone de forêt sèche et de forêt humide, FranKIE (1975), puis FRANKIE et al. (1983) ont montré que les Apoïdes solitaires appartenant aux genres Xylocopa et Anthophora butinent les fleurs des végétaux de grandes dimensions, arbres en particulier, alors que les Megachile ne visitent que les fleurs de la strate herbacée. Dans la région de Bambey où les arbres fleuris sont rares, les abeilles modifient leur comportement en récoltant le plus possible sur les végétaux herbacés et les arbustes assez développés pour leur offrir une masse florale abondante.

\section{CONCLUSIONS ET APPLICATIONS}

Les résultats de cette étude palynologique et la connaissance des ressources florales disponibles en régions cultivées du Sénégal à la fin de la saison des pluies sont en étroite relation avec le comportement des abeilles solitaires. Ces dernières, compte tenu de la biologie originale de chaque espèce, sélectionnent parmi les végétaux, ceux qui, par les caractères de leurs fleurs, exercent une attraction qui satisfait leurs besoins. Ce choix a certainement une influence directe sur la dynamique de la végétation spontanée et rudérale par leur action pollinisatrice évidente dans la plupart des cas.

Cette adaptation peut être mise à profit pour améliorer la pollinisation et donc le rendement en graines de plantes cultivées. Il serait intéressant par exemple de favoriser la nidification du genre Tetralonia à proxi- mité des champs d'I. batatas, espèce préférentiellement pollinisée par cet insecte.

Les Anthophora et les Xylocopa, sont les uns et les autres attirés par les dialypétales zygomorphes à calice glanduleux discifère d'une part, et par les gamopétales appartenant aux Asteridae notamment, à fleurs solitaires avec un tube de large diamètre ou sinon à fleurs groupées en inflorescences denses et à tube court et étroit d'autre part. Ils ont une langue très longue. Cette dernière leur permet d'absorber le nectar situé dans le fond des corolles et de récolter le pollen de ces fleurs dont la plupart des étamines sont latérales ou situées sur le pétale inférieur.

Il serait aisé d'utiliser ces aptitudes en favorisant la nidification de ces deux genres d'insectes à proximité des champs de tournesol. Il conviendrait d'aménager des talus et des haies de ligneux dont les fleurs ne font pas l'objet de récoltes polliniques ou nectarifères par les Apoïdes solitaires, donc en éliminant les Leguminosae telles que le genre Cassia, et tout particulièrement C. siamea Lam. Ce dernier pourrait être avantageusement remplacé par du Nerium oleander, le laurier rose, ou un autre ligneux à tige creuse ou à moelle abondante.

La forte attractivité des espèces du genre Megachile par les Leguminosae (Papilionaceae) est actuellement bien connue dans tous les pays des régions tempérées de l'hémisphère nord. Elle se retrouve de façon identique en régions tropicales. Sous ces climats, un tel attrait pour les Leguminosae pourrait être utilisé pour favoriser, au sein de cette famille, la pollinisation des Papilionaceae d'intérêt économique comme les Vigna notamment.

Recu le 19 décembre 1985 Accepté le 31 décembre 1986.

\section{REMERCIEMENTS}

Les auteurs expriment toute leur gratitude à R. DESMIER DE CHENON pour la communication des plantes de son herbier à des fins d'analyses morphologiques comparatives des pollens, ainsi qu'à M. H. Merlier et J.-P. Lebrun, pour les identifications des végétaux sur lesquels les insectes ont été recueillis.

\section{RÉFÉRENCES BIBLIOGRAPHIQUES}

Baijnath H., Ramcharum S., 1983. Aspects of pollination and floral development in Ficus capensis Thurb. (Moraceae). Bothalia, 14 (3-4), 883-888.

Baker H. G., Baker I., 1983. Floral nectar constituents in relation to pollinator type. In Jones C. E., Little R. J. : Handbook of Experimental Pollination Biology, Van Nostrand Reinhold Co., New York, Scarborough, Melbourne, Wokingham, 558 p., 117-141.

Buchman S. L., 1983. Buzzpollination in Angiosperms. In Jones C. E., Little R. J. : Handbook of Experimental Pollination Biology, Van Nostrand Reinhold Co., New York, Scarborough, Melbourne, Workingham, 558 p., 73-113.

Crane E., Walker P., Day R., 1984. Directory of important honey sources. I.B.R.A. ed., London, 384 p.

Cronquist A., 1968. The evolution and classification of flowering plants. Thomas Nelson ed., London, $396 \mathrm{p}$.

Dobson H. E. M., 1984. Pollen lipids in bee-visited flowers. $5^{\mathrm{e}}$ Symp. Intern., Pollination, Versailles, 27-30 sept. 1983. Les Colloques de l'I.N.R.A., 21, 61-64.
Dyer J. G., Skinn A. F., 1978. Pollen collected by Calliopsis andreniformis Smith in North America (Hym., Andrenidae). J. Kans. entomol. Soc., 51, 787-795.

Erdtman G., 1952. Pollen morphology and plant taxonomy. Angiosperms. An introduction to palynology I. Almqvist \& Wiksell, Stockholm, $539 \mathrm{p}$.

Erickson E., Buchman S. L., 1983. Electrostatics and pollination. In Jones C. E. \& Little R. J. : Handbook of Experimental Pollination Biology, Van Nostrand Reinhold Co., New York, Scarborough, Melbourne, Wokingham, 558 p., 173-194.

Faegri K., Van Der Pijl L., 1979. The principles of pollination ecology. Pergamon Press, Oxford, New York, Toronto, Sydney, Paris, Frankfurt, $3^{\mathrm{e}}$ édition, $244 \mathrm{p}$.

Frankie G. W., 1975. Tropical forest phenology and pollinator plant coevolution. In Gilbert L. E., Raven P. H. : Coevolution of Animals and Plants, 2 de édition, 246 p., 192-209.

Frankie G. W., Haber W. A., 1983. Why bees move among massflowering neo-tropical trees. In Jones C. E., Little R. J. : Handbook 
of Experimental pollination biology, Van Nostrand Reinhold Co., New York, Scarborough, Melbourne, Wokingham, 558 p., 360-372.

Frankie G. W., Haber W. A., Opler P. A., Bawa K. S., 1983. Characteristics and organisation of the large bee pollination system in the Costa Rica dry forest. In Jones C. E., Little R. J. : Handbook of Experimental pollination biology, Van Nostrand Reinhold Co., New York, Scarborough, Melbourne, Wokingham, 558 p., 411-447.

Gillon Y., 1986. Coévolution cumulative et coévolution substitutive. Acta Oecologia, Oecol. Gener., 7 (1), 27-36.

Hesse M., 1981. The fine structure of the exine in relation to the stickiness of Angiosperm pollen. Rev. Palaeobot Palynol., 35, 8192.

Hurd P. D., 1978. An annoted catalog of the Carpenter Bees (genus Xylocopa Latreille) of the Western Hemisphere (Hymenoptera, Anthophoridae), Inst. Press, Washington DC., $106 \mathrm{p}$.

Hou D., 1969. Pollen of Sarawakodendron (Celastraceae) and some related genera, with notes on techniques. Blumea, 17, 97-120.

Jermy T., 1984. Evolution of insect/host plant relationships. $A m$. Nat., 124, 609-630.

Kalin Arroyo M. T., 1981. Breeding systems and pollination biology in Leguminosae. In Polhill R. M., Raven P. H. : Advances in Legume Systematics, London, 723-769.

Lachaise D., 1982. Comment les peuplements de plantes et d'insectes phytophages se façonnent mutuellement : la théorie de la coévolution de la structure des peuplements. Rev. Ecol. (Terre Vie), 36, 481-538.

Lobreau-Callen D., 1983a. Analyse de la répartition géographique des Malpighiaceae d'après les caractères du pollen et de la pollinisation. Bothalia, 14 (3-4), 871-881.

Lobreau-Callen D., 1983b. Insectes pollinisateurs et pollen des fleurs à élaiophores. Bull. Mus. natn. Hist. nat., Paris, $4^{\mathrm{e}}$ sér., 5, sect. B, Adansonia, 2, 205-212.
Lobreau-Callen D., Callen G., 1982. Quelle est la composition pollinique d'un miel exotique, I. Bull. Soc. vers. Sci. nat., sér. 4, 9, 7085 .

Lobreau-Callen D., Coutin R., 1984. Pollens et Apoïdes du Sénégal : pollinisation et comportement. 5e Symp. internat. « Pollinisation "), Versailles, sept. 1983, Les colloques de l'I.N.R.A., 21, $267-$ 273.

McGregor S. E., 1976. Insect pollination of cultivated crop plants. Agriculture Handbook, 496, 411 p.

Merlier H., Montégut J., 1982. Adventices tropicales. Flore aux stades plantule et adulte de 123 espèces africaines ou pantropicales. Groupe de travail " désherbage des cultures tropicales », GERDAT, Ministère des Relations extérieures - Coopération et Développement éditeur, 490 p., 195 pl.

Praglowski J., Grafstroem E., 1980. The pollen morphology of the tribe Calenduleae with reference to taxonomy. Bot. Notiser, 133, $177-188$.

Schmidt J. O., 1985. Phagostimulants in pollen. J. apic. Res., 24, 107-114.

Southwick E. E., Loper G. M., Sadwick S. E., 1981. Nectar production, composition, energetics and pollinator attractiveness in spring flowers of western New York. Am. J. Bot., 68, 994-1002.

Tasei J. N., 1977. Possibilité de multiplication du pollinisateur de la Luzerne, Megachile pacifica Panz. en France. Apidologie, 8, 61-82. Vogel S., 1974. Oelblumen und ölsammelnde Bienen. Trop. subtrop. Pflanzenwelt, 7, 283-547.

Wilmer P. G., 1980. The effects of insect visitors on nectar constituents in temperate plants. Oecologia (Berl.), 47, 270-277.

Zandonella P., Dumas C., Gaude T., 1981. Sécrétions dans la pollinisation et la fécondation : revue des données récentes. Apidologie, 12, 383-396. 